\title{
A homology and cohomology theory for real projective varieties
}

\author{
Jyh-Haur Teh
}

July 1, 2018

\begin{abstract}
In this paper we develop homology and cohomology theories which play the same role for real projective varieties that Lawson homology and morphic cohomology play for projective varieties respectively. They have nice properties such as the existence of long exact sequences, the homotopy invariance, the Lawson suspension property, the homotopy property for bundle projection, the splitting principle, the cup product, the slant product and the natural transformations to singular theories. The Friedlander-Lawson moving lemma is used to prove a duality theorem between these two theories. This duality theorem is compatible with the $\mathbb{Z}_{2}$-Poincaré duality for real projective varieties with connected full real points.
\end{abstract}

\section{Introduction}

The study of solving polynomial equations dates back to the very beginning of mathematics. Finding general algebraic solutions of a given equation was the original goal. This goal was achieved for equations of degree 2, 3 and 4 . But it was proved by Abel and Galois that it was impossible for equations of degree 5. Galois theory was created several decades later to study some properties of the roots of equations. At the same time, people started to consider the more complicated problem of solving polynomial equations of more than one variable. The zero loci of polynomial equations, which are called algebraic varieties, are basic source of geometry and exemplify many important geometric phenomena. In this paper, we study the properties of projective varieties which are the zero loci of homogeneous polynomials in projective spaces.

Algebraic cycles are some finite formal sum of irreducible subvarieties with integral coefficients. The group $Z_{p}(X)$ of $p$-cycles of a projective variety $X$ encodes many properties of $X$. For many decades, since $Z_{p}(X)$ is a very large group in general, quotients of $Z_{p}(X)$ were studied instead. For example, the quotient of $Z_{p}(X)$ by rational equivalence is the Chow group $C H_{p}(X)$ on which the intersection theory of algebraic varieties can be built [12. 
For a complex projective variety $X$, the group $Z_{p}(X)$ has additional natural structure. According to the Chow theorem, there is a canonical way, by means of Chow forms, to give $\mathscr{C}_{p, d}(X)$, the set of effective $p$-cycles of degree $d$, the structure of a projective variety. Thus $\mathscr{C}_{p, d}(X)$ has a canonical analytic topology. Since $Z_{p}(X)$ is the group completion of the monoid $\prod_{d>0} \mathscr{C}_{p, d}(X)$, it inherits a topology from this analytic structure which makes it a topological group. From this point of view, methods from topology, especially from homotopy theory, come into play. The title of the Hirzebruch's book, "Topological methods in algebraic geometry" [14], perfectly describes the way we study projective varieties via their cycle groups. The starting point of this approach is the Lawson suspension theorem [15, which says that there is a homotopy equivalence between $Z_{p}(X)$ and $Z_{p+1}(\not X X)$, where $\not{Z} X$ is the projective cone over $X$ (or more topologically the Thom space of the $\mathcal{O}(1)$ bundle over $X$ ). It is then natural to define the Lawson homology group $L_{p} H_{n}(X)=\pi_{n-2 p} Z_{p}(X)$, the $(n-2 p)$-th homotopy group of $Z_{p}(X)$. When $p=0$, we have by the Dold-Thom theorem that $L_{0} H_{n}(X)=H_{n}(X ; \mathbb{Z})$ (the singular homology of $X$ ). Thus we view the Lawson homology as an enrichment of singular homology for projective varieties. This point of view will be strengthened after we develop a corresponding theory for real projective varieties and extend some classical theorems, for example the Harnack-Thom theorem [25], from singular homology.

In the past 15 years, Lawson, Friedlander, Mazur, Gabber, Michelsohn, Lam, Lima-Filho, Walker and dos Santos have discovered many properties of Lawson homology and have related it to other theories like Chow groups, higher Chow groups, motivic cohomology and algebraic $K$-theory. Notably, morphic cohomology was established by Friedlander and Lawson, and a duality theorem between morphic cohomology and Lawson homology was proved by using their moving lemma [6], 7]. It has been shown that Lawson homology and morphic cohomology groups admit limit mixed Hodge structure in [10, 6] and [24.

Algebraic topology also reaps the bounty of this harvest. Since

$$
Z_{0}\left(A^{n}\right)=\frac{Z_{0}\left(\mathbb{P}^{n}\right)}{Z_{0}\left(\mathbb{P}^{n-1}\right)}=K(\mathbb{Z}, 2 n)
$$

where $K(\mathbb{Z}, 2 n)$ is the Eilenberg-Mac Lane space, we are able to represent many Eilenberg-Mac Lane spaces by more concrete algebraic cycle spaces. For example, consider the natural embedding $\mathscr{G}^{q}\left(\mathbb{P}^{n}\right) \subset \mathscr{C}^{q}\left(\mathbb{P}^{n}\right)$ of the Grassmannian of codimension-q planes into the limit space $\mathscr{C}^{q}\left(\mathbb{P}^{n}\right)=\lim _{d \rightarrow \infty} \mathscr{C}_{d}^{q}\left(\mathbb{P}^{n}\right)$ of codimension-q cycles of $\mathbb{P}^{n}$. Letting $n$ go to infinity, we get a map

$$
B U_{q} \stackrel{c}{\longrightarrow} K(\mathbb{Z}, 2) \times K(\mathbb{Z}, 4) \times \cdots \times K(\mathbb{Z}, 2 q)
$$

where $B U_{q}=\lim _{n \rightarrow \infty} \mathscr{G}^{q}\left(\mathbb{P}^{n}\right)$ is the classifying space for the unitary group $U_{q}$. A beautiful theorem by Lawson and Michelsohn [21] says that for each $q \geq 1$, this map induces an isomorphism

$$
\mathbb{Z} \cong \pi_{2 q}(B U) \stackrel{c_{*}}{\longrightarrow} \pi_{2 q}(K(\mathbb{Z}, e v)) \cong \mathbb{Z}
$$


which is multiplication by $(q-1)$ ! where $K(\mathbb{Z}, e v)=\prod_{i=1}^{\infty} K(\mathbb{Z}, 2 i)$. Our next example is from morphic cohomology.

- There is a join pairing in algebraic cocycle groups which induces the cup product in singular cohomology.

- The Chern classes in morphic cohomology map to the Chern classes in singular cohomology.

- The cocycle group $Z^{m}(X)$ of a $m$-dimensional smooth projective manifold is homotopy equivalent to the mapping space $\operatorname{Map}(X, K(\mathbb{Z}, 2 m))$.

The Fundamental Theorem of Algebra says that a degree $d$ polynomial in one variable over $\mathbb{C}$ has $d$ zeros in complex plane counting multiplicities. A polynomial $f$ of degree $d$ over $\mathbb{R}$ needs not have $d$ real zeros, but if we take $Z(f)$ to be the set of $d$ zeros of $f$, and $Z(f)^{a v}$ to be the set of nonreal zeros of $f$ counting multiplicities, we find that if we define $R(f)=Z(f)-Z(f)^{a v}$, since nonreal zeros appear in conjugate pairs, the cardinality $|R(f)|$ of $R(f)$ and the degree $d$ have the following relation:

$$
|R(f)| \equiv d \bmod 2
$$

This is the Reduced Real Fundamental Theorem of Algebra.

The principle is that everything in the complex world has a counterpart in the real world with $\mathbb{Z}_{2}$-coefficients. Over $\mathbb{C}$, the utility of (co)homology theory with $\mathbb{Z}$-coefficients is related to the fact that the zeros of any polynomial can be counted. But over $\mathbb{R}$, we can only count the zeros modulo 2 . The bridge from the complex world to the real world passes to a quotient of the set of elements invariant under conjugation. The reduced real cycle group is defined as

$$
R_{p}(X)=\frac{Z_{p}(X)_{\mathbb{R}}}{Z_{p}(X)^{a v}}
$$

where $X$ is a real projective variety, $Z_{p}(X)_{\mathbb{R}}$ is the set of $p$-cycles which are invariant under conjugation and $Z_{p}(X)^{a v}$ are cycles of the form $c+\bar{c}$. This group first appeared in the thesis of Lam where he proved the Lawson suspension theorem for reduced real cycle groups. We develop this idea further to get a homology-like theory called reduced real Lawson homology and a cohomologylike theory called reduced real morphic cohomology. In section 2, we define the topology on some cycle groups. In section 3, we develop relative theories of reduced real Lawson homology and reduced real morphic cohomology, and get some long exact sequences. The functoriality properties of reduced real theories are considered in section 4 . We show in section 5 that the reduced real morphic cohomology has many nice properties: the homotopy invariance, the splitting principle, the suspension property, and the homotopy property. In section 6, by using the moving lemma of Friedlander and Lawson, we prove a duality theorem between reduced real Lawson homology and reduced real morphic cohomology. As in morphic cohomology, the join pairing of cycles 
induces a cup product in the reduced real morphic cohomology and through a natural tranformation to singular cohomology with $\mathbb{Z}_{2}$-coefficients it becomes the usual cup product. The natural transformations and the $s$-maps in these two theories define a filtration in the singular homology and cohomology with $\mathbb{Z}_{2}$-coefficients respectively. Under the natural transformations constructed in section 7, we show that our duality is compatible with the usual Poincaré duality with $\mathbb{Z}_{2}$-coefficients. In appendix, we show that all cycle groups that we deal with in this paper are CW-complexes.

\section{Topology Of Reduced Real Cycle And Cocy- cle Spaces}

Throughout this paper, $X, Y$ are projective varieties of dimension $m$ and $n$ respectively and $X$ is normal. An $r$-cycle on $X$ is a linear combination of irreducible subvarieties of the same dimension $r$ in $X$ with $\mathbb{Z}$-coefficients. Let $\mathscr{C}_{r, d}(X)$ be the set of degree $d r$-cycles of $X$ with positive coefficients. By Chow theorem, $\mathscr{C}_{r, d}(X)$ has the structure of a projective variety. Thus we may consider $\mathscr{C}_{r, d}(X)$ as a complex projective variety with the analytic topology. Let $K_{r, d}(X)=\prod_{d_{1}+d_{2} \leq d}\left(\mathscr{C}_{r, d_{1}}(X) \times \mathscr{C}_{r, d_{2}}(X)\right) / \sim$ where the equivalence relation $\sim$ is given by $\left(a_{1}, b_{1}\right) \sim\left(a_{2}, b_{2}\right)$ if and only if $a_{1}+b_{2}=a_{2}+b_{1} . K_{r, d}(X)$ inherits topology from this quotient which makes $K_{r, d}(X)$ a compact Hausdorff space. And from the filtration,

$$
K_{r, 0}(X) \subset K_{r, 1}(X) \subset \cdots=Z_{r}(X),
$$

we give the space of $r$-cycles $Z_{r}(X)$ on $X$ the weak topology which means that a subset $C \subset Z_{r}(X)$ is closed if and only if $C \cap K_{r, d}(X)$ is closed for all $d$. This topology makes $Z_{r}(X)$ a topological abelian group. In general, let

$$
A_{1} \subset A_{2} \subset A_{3} \subset \cdots=A
$$

be a chain of closed inclusions of topological spaces. We define the topology on $A$ by declaring a subset $C \subset A$ to be closed if and only if its intersection $C \cap A_{i}$ is closed for all $i \geq 1$. This topology is called the weak topology with respect to the subspaces.

Definition Let $\mathscr{C}_{r}(Y)(X)$ be the topological submonoid of $\mathscr{C}_{r+m}(X \times Y)$ consisting of elements which are equidimensional of relative dimension $r$ over $X$. Let $Z_{r}(Y)(X)$ be the naive group completion $\left[\mathscr{C}_{r}(Y)(X)\right]^{+}$of $\mathscr{C}_{r}(Y)(X)$ with the quotient topology. We call $Z_{r}(Y)(X)$ the dimension $r$ cocycle group of $X$ with values in $Y$. Let $\mathscr{M} \operatorname{or}\left(X, \mathscr{C}_{r}(Y)\right)=\coprod_{d \geq 0}^{\infty} \mathscr{M} \operatorname{or}\left(X, \mathscr{C}_{r, d}(Y)\right)$ be the space of all morphisms from $X$ to $\mathscr{C}_{r}(Y)$ with the compact-open topology. Let $\mathscr{M} \operatorname{or}\left(X, Z_{r}(Y)\right)=\left[\mathscr{M} \operatorname{or}\left(X, \mathscr{C}_{r}(Y)\right)\right]^{+}$be the naive group completion of $\mathscr{M}$ or $\left(X, \mathscr{C}_{r}(Y)\right)$. 
By the graphing construction of Friedlander and Lawson [8, Theorem 1.2], there is a topological group isomorphism

$$
\mathcal{G}: \mathscr{M} \operatorname{or}\left(X, Z_{r}(Y)\right) \rightarrow Z_{r}(Y)(X)
$$

Definition A real projective variety $V \subset \mathbb{C P}^{n}$ is a complex projective variety which is invariant under the conjugation of $\mathbb{C P}^{n}$. A subvariety $V \subset X \times Y$ is real if and only if $\bar{V}=V$ where $\bar{V}=\{(\bar{x}, \bar{y}) \mid(x, y) \in V\}$. A cycle on $V$ is real if all its components are real subvarieties of $V$. A cycle $c$ is averaged if $c=a+\bar{a}$ for some cycle $a$.

We will write the conjugate of a point $x \in X$ as $\bar{x}$. The conjugation induces a map on cycle groups. For $f \in Z_{r}(Y)(X)$, define $\bar{f}(x)=\overline{f(\bar{x})}$. This notation is convenient when we think of $f$ as a map from $X$ to the cycle space of $Y$.

Proposition 2.1 For $f \in Z_{r}(Y)(X), f$ is real if and only if $\bar{f}=f$.

Proof Since $f$ is real, the set $\{(x, f(x)) \mid x \in X\}$ is equal to the set $\{(\bar{x}, \overline{f(x)}) \mid x \in$ $X\}$. The point $(\bar{x}, f(\bar{x}))$ is in this set and $f$ is a function, hence $f(\bar{x})=\overline{f(x)}$. Therefore $\bar{f}=f$. Another direction is trivial.

Definition The filtration

$$
K_{r, 0}(X) \subset K_{r, 1}(X) \subset K_{r, 2}(X) \subset \cdots=Z_{r}(X)
$$

is called the canonical $r$-filtration of $X$ and if $X$ is a real projective variety,

$$
K_{r, 0}(X)_{\mathbb{R}} \subset K_{r, 1}(X)_{\mathbb{R}} \subset K_{r, 2}(X)_{\mathbb{R}} \subset \cdots=Z_{r}(X)_{\mathbb{R}}
$$

is called the canonical real $r$-filtration of $X$ where $K_{r, i}(X)_{\mathbb{R}}$ is the subset of real cycles in $K_{r, i}(X)$ and

$$
K_{r, 0}(X)^{a v} \subset K_{r, 1}(X) \subset K_{r, 2}(X)^{a v} \subset \cdots=Z_{r}(X)^{a v}
$$

is called the canonical averaged $r$-filtration of $X$ where $K_{r, i}(X)^{a v}$ is the subset of averaged cycles in $K_{r, i}(X)$. If a filtration is defined by a sequence of compact sets, this filtration is called a compactly filtered filtration.

Definition Let $Z_{r}(X)_{\mathbb{R}}$ be the subgroup of $Z_{r}(X)$ consisting of real cycles and let $Z_{r}(X)^{a v}=\left\{V+\bar{V} \mid V \in Z_{r}(X)\right\}$ be the averaged cycle group. We give $Z_{r}(X)_{\mathbb{R}}$ and $Z_{r}(X)^{a v}$ the subspace topology of $Z_{r}(X)$ which are same as the weak topology defined by the canonical real $r$-filtration and the canonical averaged $r$-filtration respectively.

Proposition 2.2 Suppose that $Y$ is a subvariety of a projective variety $X$. Then $Z_{r}(Y)$ is a closed subgroup of $Z_{r}(X)$. If $Y \subset X$ are real projective varieties, then $Z_{r}(Y)_{\mathbb{R}}, Z_{r}(Y)^{a v}$ are closed subgroups of $Z_{r}(X)_{\mathbb{R}}$ and $Z_{r}(X)^{a v}$ respectively. 
Proof This is because $Z_{r}(Y) \cap K_{r, d}(X)=K_{r, d}(Y)$ which is closed in $K_{r, d}(X)$. Similarly, the closedness follows from the fact $Z_{r}(Y)_{\mathbb{R}} \cap K_{r, d}(X)_{\mathbb{R}}=K_{r, d}(Y)_{\mathbb{R}}$ and $Z_{r}(Y)^{a v} \cap K_{r, d}(X)^{a v}=K_{r, d}(Y)^{a v}$.

Let $\mathscr{C}_{r, d}(Y)(X)$ be the subspace of $\mathscr{C}_{r+m, d}(X \times Y)$ consisting of cycles which are equidimensional over $X$. It is shown in [6, Lemma 1.4] that $\mathscr{C}_{r, d}(Y)(X)$ is a Zariski open set of $\mathscr{C}_{r+m, d}(X \times Y)$. Let $K_{r, d}(Y)(X)=\coprod_{d_{1}+d_{2} \leq d} \mathscr{C}_{r, d_{1}}(Y)(X) \times$ $\mathscr{C}_{r, d_{2}}(Y)(X) / \sim$ with the quotient topology, and $K_{r, d}(Y)(X)_{\mathbb{R}}, K_{r, d}(Y)(X)^{a v}$ the subspaces of $K_{r, d}(Y)(X)$ consisting of elements which can be represented by real cycles and averaged cycles respectively. Then we may formed the corresponding canonical filtration, canonical real filtration and canonical averaged filtration.

Definition Suppose that $X, Y$ are real projective varieties. Let

$$
\begin{gathered}
Z_{r}(Y)(X)_{\mathbb{R}}=Z_{r}(Y)(X) \cap Z_{r+m}(X \times Y)_{\mathbb{R}} \\
Z_{r}(Y)(X)^{a v}=Z_{r}(Y)(X) \cap Z_{r+m}(X \times Y)^{a v}
\end{gathered}
$$

with the subspace topology of $Z_{r}(Y)(X)$. Since the topology of $Z_{r}(Y)(X)$ is given by the weak topology from the canonical filtration, the topology of $Z_{r}(Y)(X)_{\mathbb{R}}$ and $Z_{r}(Y)(X)^{a v}$ are given by the weak topology of the canonical real and averaged filtration respectively.

Definition A filtration of a topological space $T$ by a sequence of subspaces

$$
T_{0} \subset T_{1} \subset \cdots \subset T_{j} \cdots=T
$$

is said to be locally compact if for any compact subset $K \subset T$, there exists some $e \geq 0$ such that $K \subset T_{e}$.

Lemma 2.3 Suppose that $X$ is a Hausdorff space and the topology on $X$ is given by the filtration

$$
T_{0} \subset T_{1} \subset \cdots \subset T_{j} \cdots=X
$$

by the weak topology. Then this filtration is a locally compact filtration.

Proof Let $K \subset X$ be a compact subset. Assume that $K$ is not contained in any $T_{e}$, then we can find a sequence $\left\{x_{i}\right\}_{i \in I}$ where $x_{i} \in\left(T_{i}-T_{i-1}\right) \cap K$ and $I$ is a sequence of distinct integers which goes to infinity. Let $U_{k}=X-\left\{x_{i}\right\}_{i \in I, i \neq k}$. Then $U_{k} \cap T_{j}=T_{j}-\left\{x_{i} \mid i \leq j, i \neq k\right\}$ which is open for all $j$. Thus $U_{k}$ is an open subset of $X$ and $K \subset \cup_{i \in I} U_{k}$ but we are unable to find a finite subcover which contradicts to the compactness of $K$. So $K$ has to be contained in some $T_{e}$.

From this lemma, we know that all of our canonical filtrations are locally compact. 
Proposition 2.4 For real projective varieties $X, Y, Z_{r}(Y)(X)_{\mathbb{R}}$ and $Z_{r}(Y)(X)^{a v}$ are closed subgroups of $Z_{r}(Y)(X)$. In particular, $Z_{r}(Y)_{\mathbb{R}}$ and $Z_{r}(Y)^{\text {av }}$ are closed subgroups of $Z_{r}(Y)$.

Proof Define $\psi: Z_{r}(Y)(X) \longrightarrow Z_{r}(Y)(X)$ by $\psi(f)=\bar{f}-f$. Since $\psi$ is continuous and $Z_{r}(Y)(X)_{\mathbb{R}}$ is the kernel of $\psi, Z_{r}(Y)(X)_{\mathbb{R}}$ is closed. Suppose that $\left\{f_{n}+\overline{f_{n}}\right\}$ is a sequence in $Z_{r}(Y)(X)^{a v}$ which converges to $c$. Since the canonical averaged $r$-filtration of $X$ is locally compact, by Lemma 2.3, $A=$ $\left\{f_{n}+\overline{f_{n}}\right\} \cup\{c\}$ is contained in $K_{r, d}(Y)(X)^{a v}$ for some $d>0$. Therefore there exist $g_{n} \in K_{r, d}(Y)(X)^{a v}$ such that $g_{n}+\overline{g_{n}}=f_{n}+\overline{f_{n}}$ for all $n$. Since the sequence $\left\{g_{n}\right\} \subset K_{r+m, d}(X \times Y)$ and $K_{r+m, d}(X \times Y)$ is compact, hence $\left\{g_{n}\right\}$ has a convergent subsequence, which we denote by $\left\{g_{n_{i}}\right\}$. Suppose that $\left\{g_{n_{i}}\right\}$ converges to a cycle $g$. Then $\left\{\overline{g_{n_{i}}}\right\}$ converges to $\bar{g}$. Therefore $g_{n_{i}}+\overline{g_{n_{i}}}$ converges to $g+\bar{g}$ which implies $c=g+\bar{g}$. Thus $Z_{r}(Y)(X)^{a v}$ is closed. Take $X$ to be a point, it follows immediately that $Z_{r}(Y)_{\mathbb{R}}$ and $Z_{r}(Y)^{a v}$ are closed in $Z_{r}(Y)$.

Definition Suppose that $X, Y$ are real projective varieties. We define the reduced real cycle group to be

$$
R_{r}(X)=\frac{Z_{r}(X)_{\mathbb{R}}}{Z_{r}(X)^{a v}},
$$

and the reduced real $Y$-valued cocycle group to be

$$
R_{r}(Y)(X)=\frac{Z_{r}(Y)(X)_{\mathbb{R}}}{Z_{r}(Y)(X)^{a v}}
$$

Furthermore, if $Y$ is a real subvariety of $X$. We define

$$
Z_{r}(X, Y)^{a v}=\frac{Z_{r}(X)^{a v}}{Z_{r}(Y)^{a v}},
$$

and

$$
Z_{r}(X, Y)_{\mathbb{R}}=\frac{Z_{r}(X)_{\mathbb{R}}}{Z_{r}(Y)_{\mathbb{R}}} .
$$

All these groups are enrolled with quotient topology.

A topological group $G$ is a group which is also a Hausdorff space and for $(g, h) \in G \times G$, the product $(g, h) \longrightarrow g h^{-1}$ is a continuous map. From the Proposition above, we have the following result.

Corollary 2.5 For real projective varieties $X, Y, R_{r}(Y)(X)$ and $R_{r}(X)$ are topological abelian groups.

Proof This is from the general fact that the quotient of a topological group by any of its closed normal subgroups is a topological group. 
The homotopy types of $Z_{r}\left(\mathbb{P}^{n}\right)_{\mathbb{R}}$ and $Z_{r}\left(\mathbb{P}^{n}\right)^{a v}$ were computed in [19] which are quite complicated but the homotopy type of $R_{r}\left(\mathbb{P}^{n}\right)$ is much simpler. In the following we will see that the reduced real cycle groups are closely related to the singular homology with $\mathbb{Z}_{2}$-coefficients of the real points. These are some of the reasons that we work on the reduced real cycle groups.

There are two natural ways to put topology on $R_{r}(X)$. One is the weak topology from the filtration induced by the canonical real filtration on $R_{r}(X)$ another one is the quotient topology. We show that these topologies coincide on $R_{r}(X)$.

Proposition 2.6 Suppose that $G$ is a topological group and

$$
K_{1} \subset K_{2} \subset \cdots=G
$$

is a filtration filtered by compact subsets of $G$ which generates the topology of $G$. Let $H$ be a closed normal subgroup of $G$ and $q: G \longrightarrow G / H$ be the quotient map. Denote the restriction of $q$ to $K_{k}$ by $q_{k}$ and $M_{k}=q\left(K_{k}\right)$. We define a topology on $M_{k}$ by making $q_{k}$ a quotient map, for each $k$. Then

1. $M_{k}$ is a subspace of $M_{k+1}$ for all $k$.

2. the weak topology of $G / H$ defined by the filtration

$$
M_{1} \subset M_{2} \subset \cdots=G / H
$$

coincides with the quotient topology of $G / H$.

Proof 1. If $C \subset M_{k+1}$ is a closed subset, since $M_{k+1}$ is compact, $C$ is also compact. From the commutative diagram,

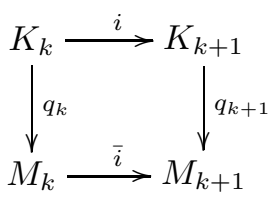

we have $\bar{i}^{-1}(C)=q_{k} i^{-1} q_{k+1}^{-1}(C)$ which is compact thus closed. So $\bar{i}$ is continuous. Since $M_{k}$ is compact and $\bar{i}$ is injective, it is an embedding.

2. Let $C$ be a closed subset of $G / H$ under the quotient topology. Then $q^{-1}(C)$ is closed which means $q^{-1}(C) \cap K_{k}$ is closed for all $k . q_{k}^{-1}(C \cap$ $\left.M_{k}\right)=q^{-1}(C) \cap K_{k}$ is closed, so $C \cap M_{k}$ is closed for all $k$ thus $C$ is closed under the weak topology. On the other hand, assume that $C$ is a closed subset of $G / H$ under the weak topology, that is, $C \cap M_{k}$ is closed for all $k$. $q^{-1}(C) \cap K_{k}=q^{-1}\left(C \cap M_{k}\right) \cap K_{k}=q_{k}^{-1}\left(C \cap M_{k}\right)$ which is closed for all $k$, thus $C$ is a closed subset in the quotient topology.

Corollary 2.7 Suppose that $X$ is a real projective variety and $Y$ is a real subvariety of $X$. Consider the canonical real and averaged filtrations of $X$ :

$$
K_{r, 1}(X)_{\mathbb{R}} \subset K_{r, 2}(X)_{\mathbb{R}} \subset \cdots=Z_{r}(X)_{\mathbb{R}}
$$




$$
K_{r, 1}(X)^{a v} \subset K_{r, 2}(X)^{a v} \subset \cdots=Z_{r}(X)^{a v}
$$

and the quotient maps $q_{1}: Z_{r}(X)_{\mathbb{R}} \longrightarrow R_{r}(X), q_{2}: Z_{r}(X)_{\mathbb{R}} \longrightarrow Z_{r}(X, Y)_{\mathbb{R}}$, $q_{3}: Z_{r}(X)^{a v} \longrightarrow Z_{r}(X, Y)^{a v}$. Then

1. for all $k, q_{1}\left(K_{r, k}(X)_{\mathbb{R}}\right), q_{2}\left(K_{r, k}(X)_{\mathbb{R}}\right)$ and $q_{3}\left(K_{r, k}(X)^{\text {av }}\right)$ are subspaces of $q_{1}\left(K_{r, k+1}(X)_{\mathbb{R}}\right), q_{2}\left(K_{r, k+1}(X)_{\mathbb{R}}\right)$ and $q_{3}\left(K_{r, k+1}(X)^{a v}\right)$ respectively.

2. the weak topology of $R_{r}(X), Z_{r}(X, Y)_{\mathbb{R}}, Z_{r}(X, Y)^{\text {av }}$ induced from the filtrations above coincide with the quotient topology on them.

Proposition 2.8 For a real projective variety $X$, let Re $X$ denote the set of real points of $X$ and let $Z_{0}(R e X)$ denote the subgroup of $Z_{0}(X)$ generated by real points of $X$. Then

1. $Z_{0}(\operatorname{Re} X)$ is a closed subgroup of $Z_{0}(X)_{\mathbb{R}}$.

2. $R_{0}(X)$ is isomorphic as a topological group to $\frac{Z_{0}(\operatorname{ReX})}{2 Z_{0}(\operatorname{ReX})}$.

Proof 1. Since $X$ is compact, the quotient map from $X \times \cdots \times X$ (k-times) to the symmetric product $S P^{k}(X)$ is a closed map. $\operatorname{Re} X \times \cdots \times \operatorname{Re} X$ is a closed subset of $X \times \cdots \times X$ thus $S P^{k}(\operatorname{Re} X)$ is a closed subset of $S P^{k}(X)$. Consider the following filtrations:

$$
A_{0} \subset A_{1} \subset A_{2} \subset \cdots=Z_{0}(\operatorname{Re} X)
$$

where $A_{n}=\coprod_{k=0}^{n} S P^{k}(\operatorname{Re} X) \times S P^{k}(\operatorname{Re} X) / \sim$ and

$$
B_{0} \subset B_{1} \subset B_{2} \subset \cdots=Z_{0}(X)
$$

where $B_{n}=\coprod_{k=0}^{n} S P^{k}(X) \times S P^{k}(X) / \sim$ and $\sim$ is the equivalence relation coming from the naive group completion. The topology of $Z_{0}(\operatorname{Re} X)$ and $Z_{0}(X)$ are defined by these two filtrations respectively. Since $S P^{k}(\operatorname{Re} X)$ and $S P^{k}(\operatorname{Re} X)$ are compact, $A_{n}$ is closed in $B_{n}$. Observe that $Z_{0}(\operatorname{Re} X) \cap$ $B_{n}=A_{n}$, thus $Z_{0}(\operatorname{Re} X)$ is a closed subgroup of $Z_{0}(X)$ and hence a closed subgroup of $Z_{0}(X)_{\mathbb{R}}$.

2. Let $q: Z_{0}(X)_{\mathbb{R}} \longrightarrow R_{0}(X)$ be the quotient map and $i: Z_{0}(\operatorname{Re} X) \hookrightarrow$ $Z_{0}(X)_{\mathbb{R}}$ be the inclusion map. Then $q \circ i: Z_{0}(\operatorname{Re} X) \longrightarrow R_{0}(X)$ is a continuous map and $2 Z_{0}(R e X)$ is in the kernel, thus we get a continuous map $\psi: \frac{Z_{0}(\operatorname{Re} X)}{2 Z_{0}(\operatorname{Re} X)} \longrightarrow R_{0}(X)$. Since each class in $R_{0}(X)$ can be represented uniquely by cycle of real points modulo $2, \psi$ is bijective. Let

$$
K_{0} \subset K_{1} \subset K_{2} \subset \cdots=Z_{0}(X)_{\mathbb{R}}
$$

be the canonical real filtration and $T_{i}=K_{i} \cap Z_{0}(\operatorname{Re} X) . Z_{0}(\operatorname{Re} X)$ is a closed subset of $Z_{0}(X)_{\mathbb{R}}$ and $K_{i}$ is compact, thus $T_{i}$ is compact for all $i$. Since $\psi$ is bijective, $\psi\left(T_{i}+2 Z_{0}(\operatorname{Re} X)\right)=K_{i}+Z_{0}(X)^{a v}$ for each $i \geq 0$. For a closed $C \subset \frac{Z_{0}(\operatorname{ReX})}{2 Z_{0}(\operatorname{ReX})}, \psi(C) \cap\left(K_{i}+Z_{0}(X)^{a v}\right)=\psi\left(C \cap T_{i}+2 Z_{0}(\operatorname{Re} X)\right)$ which is closed, thus $\psi^{-1}$ is continuous. Then it is easy to see that $\psi$ is a topological group isomorphism. 
Definition Let $X$ be a real projective variety. For any $f \in Z_{p}(X)$, let $f=$ $\sum_{i \in I} n_{i} V_{i}$ be in the reduced form, i.e., $V_{i} \neq V_{j}$ if $i \neq j$. Let

$$
R P(f)=\sum_{i \in I, \overline{V_{i}}=V_{i}} n_{i} V_{i}
$$

which is called the real part of $f$. Let

$$
J=\left\{i \in I \mid V_{i} \text { is not real and } \overline{V_{i}} \text { is also a component of } f\right\}
$$

and for $i \in J$, let $m_{i}$ be the maximum value of the coefficients of $V_{i}$ and $\overline{V_{i}}$. Define the averaged part to be

$$
A P(f)=\sum_{i \in J} m_{i}\left(V_{i}+\bar{V}_{i}\right)
$$

and the imaginary part to be

$$
I P(f)=f-R P(f)-A P(f) .
$$

Then $f \in Z_{p}(X)_{\mathbb{R}}$ if and only if $I P(f)=0$.

Proposition 2.9 Suppose that $X, Y, Z$ are real projective varieties and $Y$ is a subvariety of $Z$, then

1. the inclusion map $i: Z_{r}(Y)(X) \longrightarrow Z_{r}(Z)(X)$ is a closed embedding.

2. the inclusion map $i: Z_{r}(Y)(X) \longrightarrow Z_{r}(Z)(X)$ induces a closed embedding $\bar{i}: R_{r}(Y)(X) \hookrightarrow R_{r}(Z)(X)$ for $r \geq 0$.

3. the inclusion map $i: Z_{r}(Z)^{a v} \longrightarrow Z_{r}(Z)_{\mathbb{R}}$ induces a closed embedding $\bar{i}: Z_{r}(Z, Y)^{a v} \hookrightarrow Z_{r}(Z, Y)_{\mathbb{R}}$ for $r \geq 0$.

Proof 1. Let

$$
\begin{aligned}
& G_{r, d}(Y)(X)=\coprod_{d_{1}+d_{2} \leq d} \mathscr{M} \operatorname{or}\left(X, \mathscr{C}_{r, d_{1}}(Y)\right) \times \mathscr{M} \operatorname{or}\left(X, \mathscr{C}_{r, d_{2}}(Y)\right), \\
& G_{r, d}(Z)(X)=\coprod_{d_{1}+d_{2} \leq d} \mathscr{M} \operatorname{or}\left(X, \mathscr{C}_{r, d_{1}}(Z)\right) \times \mathscr{M} \operatorname{or}\left(X, \mathscr{C}_{r, d_{2}}(Z)\right) .
\end{aligned}
$$

Let $p: \mathscr{M} \operatorname{or}\left(X, \mathscr{C}_{r}(Y)\right) \times \mathscr{M} \operatorname{or}\left(X, \mathscr{C}_{r}(Y)\right) \rightarrow Z_{r}(Y)(X)$ and $q: \mathscr{M}$ or $\left(X, \mathscr{C}_{r}(Z)\right) \times$ $\mathscr{M}$ or $\left(X, \mathscr{C}_{r}(Z)\right) \rightarrow Z_{r}(Z)(X)$ be the quotient maps. Let $G_{r, d}^{\prime}(Y)(X)=$ $p\left(G_{r, d}(Y)(X)\right), G_{r, d}^{\prime}(Z)(X)=q\left(G_{r, d}(Z)(X)\right)$ enrolled with the quotient topology. Then by the graphing construction of Friedlander and Lawson, the topology of $Z_{r}(Y)(X)$ and $Z_{r}(Z)(X)$ are same as the weak topologies induced by the filtrations formed by $G_{r, d}^{\prime}(Y)(X)$ and $G_{r, d}^{\prime}(Z)(X)$ respectively. The topology of $\mathscr{M} \operatorname{or}\left(X, \mathscr{C}_{r, d}(Z)\right)$ is the compact-open topology. Since $\mathscr{C}_{r, d}(Y)$ is a closed subspace of $\mathscr{C}_{r, d}(Z)$, it is easy to see 
that $\mathscr{M} \operatorname{or}\left(X, \mathscr{C}_{r, d}(Y)\right)$ is a closed subspace of $\mathscr{M} \operatorname{or}\left(X, \mathscr{C}_{r, d}(Z)\right)$. Consider $G_{r, d}^{\prime}(Y)(X)$ as a subset of $G_{r, d}^{\prime}(Z)(X)$, then $p^{-1}\left(G_{r, d}^{\prime}(Y)(X)\right)=$ $G_{r, d}(Y)(X)$ which is a closed subset of $G_{r, d}(Z)(X)$. Hence $G_{r, d}^{\prime}(Y)(X)$ is a closed subset of $G_{r, d}^{\prime}(Z)(X)$. Therefore $Z_{r}(Y)(X)$ is a closed subgroup of $Z_{r}(Z)(X)$.

2. Since the inclusion $Z_{r}(Y)(X) \stackrel{i}{\hookrightarrow} Z_{r}(Z)(X)$ is an embedding, the restriction

$$
i: Z_{r}(Y)(X)_{\mathbb{R}} \hookrightarrow Z_{r}(Z)(X)_{\mathbb{R}}
$$

is also an embedding. Since $Z_{r}(Y)(X)^{a v} \subset Z_{r}(Z)(X)^{a v}, i$ induces a map

$$
\bar{i}: R_{r}(Y)(X) \longrightarrow R_{r}(Z)(X) .
$$

If $f \in Z_{r}(Z)(X)^{a v} \cap Z_{r}(Y)(X)_{\mathbb{R}}$, then $R P(f)=2 g$ for some $g \in Z_{r}(Y)(X)_{\mathbb{R}}$. Thus $f=2 g+A P(f) \in Z_{r}(Y)(X)^{a v}$ and therefore $\bar{i}$ is injective.

Let $A_{0} \subset A_{1} \subset A_{2} \subset \cdots=Z_{r}(Y)(X)_{\mathbb{R}}, B_{0} \subset B_{1} \subset B_{2} \subset \cdots=$ $Z_{r}(Z)(X)_{\mathbb{R}}$ be the canonical real filtrations. Let $q_{1}, q_{2}$ be the quotient maps from $Z_{r}(Y)(X)_{\mathbb{R}}, Z_{r}(Z)(X)_{\mathbb{R}}$ to $R_{r}(Y)(X)$ and $R_{r}(Z)(X)$ respectively. Let $C_{k}=q_{1}\left(A_{k}\right), D_{k}=q_{2}\left(B_{k}\right)$ for all $k$. Since $i\left(A_{k}\right) \subset B_{k}$, $\bar{i}\left(C_{k}\right) \subset D_{k}$ and from the definition of canonical filtrations, $\bar{i}^{-1}\left(D_{k}\right) \subset C_{k}$. For any closed subset $W$ of $R_{r}(Y)(X), W \cap C_{k}$ is compact and by the injectivity of $\bar{i}, \bar{i}\left(W \cap C_{k}\right)=\bar{i}(W) \cap \bar{i}\left(C_{k}\right)=\bar{i}(W) \cap D_{k}$ which is compact and thus closed.

3. Since the inclusion $i: Z_{r}(Z)^{a v} \hookrightarrow Z_{r}(Z)_{\mathbb{R}}$ is an embedding and $i\left(Z_{r}(Y)^{a v}\right) \subset$ $Z_{r}(Y)_{\mathbb{R}}$, it induces a map $\bar{i}: Z_{r}(Z, Y)^{a v} \hookrightarrow Z_{r}(Z, Y)_{\mathbb{R}}$. If $\bar{i}\left(f+Z_{r}(Y)^{a v}\right)=$ $f+Z_{r}(Y)_{\mathbb{R}}=Z_{r}(Y)_{\mathbb{R}}$, then $f \in Z_{r}(Y)_{\mathbb{R}}$. This implies $R P(f) \in Z_{r}(Y)_{\mathbb{R}}$. But $f$ is also an averaged cycle, so $R P(f)=2 g$ for some $g \in Z_{r}(Y)_{\mathbb{R}}$ and $f=2 g+A P(f) \in Z_{r}(Y)^{a v}$. Hence, $\bar{i}$ is injective. For the rest, it is similar to the argument above.

From this Proposition, when we need to take quotient, we will abuse of notation and write $\frac{R_{r}(Z)(X)}{R_{r}(Y)(X)}$ and $\frac{Z_{r}(Z, Y)_{\mathbb{R}}}{Z_{r}(Z, Y)^{a v}}$ for the quotient of $R_{r}(Z)(X), Z_{r}(Z, Y)_{\mathbb{R}}$ by the images of $i$ in $R_{r}(Y)(X)$ and $Z_{r}(Z, Y)^{a v}$ respectively.

\section{Relative Theory And Long Exact Sequences}

In [25, Proposition 4.1], we develop a technique to produce a long exact sequence of homotopy groups from the exact sequence

$$
0 \rightarrow H \rightarrow G \rightarrow G / H \rightarrow 0
$$

where $H$ and $G$ are compactly generated and $H$ is a normal closed subgroup of $G$. We found that actually we not need to assume that $H$ and $G$ are compactly generated. We recall briefly the Milnor's construction (see [22]) and the Borel construction. 
For a topological group $G$, the $n$-th join of $G$ is the space

$$
G_{n}:=\left\{t_{1} g_{1} \oplus \cdots \oplus t_{n} g_{n} \mid \sum_{i=1}^{n} t_{i}=1, t_{i} \geq 0, g_{i} \in G, \forall i=1, \ldots, n\right\} / \sim
$$

with the Milnor's strong topology, and the equivalence relation $\sim$ identifies two elements $t_{1} g_{1} \oplus \cdots \oplus t_{n} g_{n} \sim t_{1}^{\prime} g_{1}^{\prime} \oplus \cdots \oplus t_{n}^{\prime} g_{n}^{\prime}$ if $t_{i}=t_{i}^{\prime}$ for all $i=1, \ldots, n$ and $g_{i}=g_{i}^{\prime}$ if $t_{i} \neq 0$.

We form the infinite join $E(G)$ of $G$ in the same manner, with the restriction that all but a finite number of the $t_{i}$ should vanish. Then $G$ acts freely on $E(G)$ and there exists a locally trivial universal principal $G$-bundle

$$
p: E G \rightarrow B G
$$

where $B G=E G / G$. Furthermore, all the homotopy groups of $E G$ vanish, and from the homotopy sequence induced by the fibration $p$, we see that $\pi_{k}(B G)=$ $\pi_{k-1}(G)$ for $k \geq 1$.

If $\phi: G \rightarrow G^{\prime}$ is a morphism between two topological groups, define $E(\phi)$ : $E(G) \rightarrow E\left(G^{\prime}\right)$ by

$$
E(\phi)\left(t_{1} g_{1} \oplus t_{2} g_{2} \oplus \cdots\right)=t_{1} \phi\left(g_{1}\right) \oplus t_{2} \phi\left(g_{2}\right) \oplus \cdots
$$

then $E(\phi)$ is an equivariant map and induces a map $B(\phi): B(G) \rightarrow B\left(G^{\prime}\right)$.

Definition Suppose that $G$ is a topological group acting on a topological space $X$. The Borel construction is the orbit space $B(X, G)=(X \times E G) / G$ where $E G$ is the universal bundle of $G$. Then there is a fibration

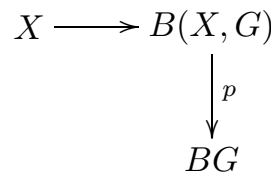

where $p: B(X, G) \longrightarrow B G$ is the projection.

Definition We say that a map $\phi: A \rightarrow B$ between two topological spaces is a weak homotopy equivalence if $\phi$ induces isomorphism $\phi_{*}: \pi_{n}(A) \rightarrow \pi_{n}(B)$ for each $n \geq 0$. We say that $A, B$ are weakly homotopy equivalent if they have the same homotopy groups.

Proposition 3.1 1. If $\phi: G \rightarrow G^{\prime}$ is a topological group homomorphism which is also a weak homotopy equivalence, then the induced map $B(\phi)$ : $B(G) \rightarrow B\left(G^{\prime}\right)$ is also a weak homotopy equivalence.

2. Let $G$ be a topological group and $\Omega B G$ be the loop space of $B G$ with based point $[e] \in B G$ where $e$ is the identity of $G$. Then there is a map $\gamma: G \rightarrow$ $\Omega B G$ which induces isomorphisms of their homotopy groups. 
3. Suppose that we have a commutative diagram of topological group homomorphisms:

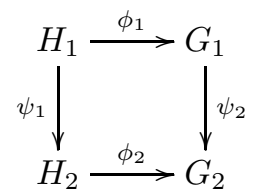

Then $\psi_{1}, \psi_{2}$ induce a map $B\left(\psi_{2}, \psi_{1}\right): B\left(G_{1}, H_{1}\right) \longrightarrow B\left(G_{2}, H_{2}\right)$. When $\psi_{1}, \psi_{2}$ are weak homotopy equivalences, $B\left(\psi_{2}, \psi_{1}\right)$ is also a weak homotopy equivalence.

4. If $\phi: G \rightarrow H$ is a morphism between topological groups and $B(\phi)_{*}$ : $B(G) \rightarrow B(H)$ is a weak homotopy equivalence. If $G$ and $H$ have the homotopy type of a $C W$-complex, then $\phi$ is a homotopy equivalence.

5. Suppose that $F: H_{1} \times I \longrightarrow H_{2}, F^{\prime}: G_{1} \times I \longrightarrow G_{2}$ are homotopies between $F_{0}=\psi_{1}, F_{1}=\psi_{1}^{\prime}$ and $F_{0}^{\prime}=\psi_{2}, F_{1}^{\prime}=\psi_{2}^{\prime}$ respectively where each $F_{t}, F_{t}^{\prime}$ are group homomorphisms. If the following diagram commutes:

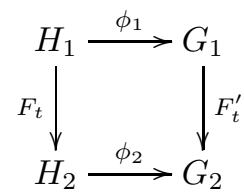

for each $t \in I$, then $B\left(\psi_{2}, \psi_{1}\right)$ is homotopic to $B\left(\psi_{2}^{\prime}, \psi_{1}^{\prime}\right)$.

Proof 1. Consider the long exact sequences on homotopy groups induced from the two fibrations

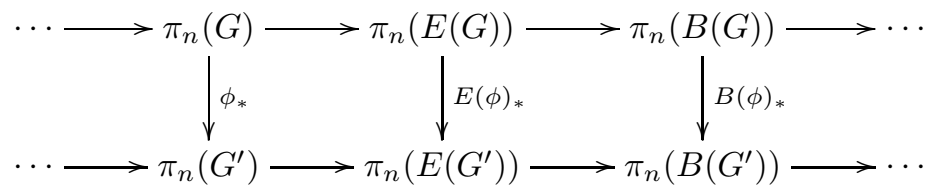

which shows that $B(\phi)_{*}$ is an isomorphism between the homotopy groups of $B(G)$ and $B\left(G^{\prime}\right)$.

2. Let $P B G$ be the path space of $B G$ with based point $[e] \in B G$. Define $\gamma: E G \rightarrow P B G$ by

$$
\gamma\left(t_{1} g_{1} \oplus t_{2} g_{2} \oplus \cdots \oplus t_{n} g_{n}\right):=\left[(1-t) e: t t_{1} g_{1}: t t_{2} g_{2}: \cdots: t t_{n} g_{n}\right]
$$

where $0 \leq t \leq 1$. Then we have a commutative diagram of two fibrations:

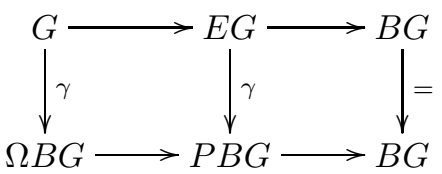


where the second row is the path fibration. Since $E G$ and $P B G$ are weakly contractible, the map $\gamma$ induces isomorphisms between the homotopy groups of $G$ and $\Omega B G$.

3. Define $B\left(\psi_{2}, \psi_{1}\right): G_{1} \times E\left(H_{1}\right) \rightarrow G_{2} \times E\left(H_{2}\right)$ by $(x, y) \rightarrow\left(\psi_{2}(x), \psi_{1 *}(y)\right)$ which induces a map $B\left(\psi_{2}, \psi_{1}\right): B\left(G_{1}, H_{1}\right) \rightarrow B\left(G_{2}, H_{2}\right)$. Consider the long exact sequences

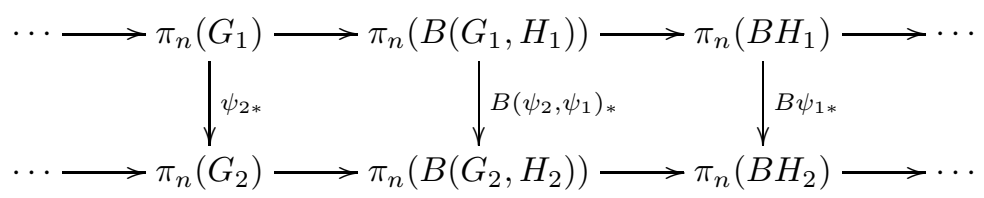

Since $\psi_{2 *}$ and $B\left(\psi_{1}\right)$ are weak homotopy equivalence, $B\left(\psi_{2}, \psi_{1}\right)$ is also a weak homotopy equivalence.

4. We have the following commutative diagram:

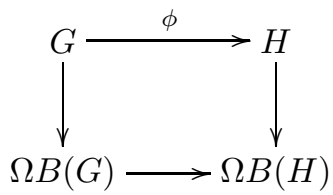

where the vertical arrows are weak homotopy equivalences. Hence the map $\phi$ induces isomorphisms of homotopy groups between $G$ and $H$. If $G$ and $H$ have the homotopy type of a CW-complex, by the Whitehead theorem, $\phi$ is a homotopy equivalence.

5. By (ii), $F$ and $F^{\prime}$ induce a map $B\left(F^{\prime}, F\right): B\left(G_{1}, H_{1}\right) \times I \longrightarrow B\left(G_{2}, H_{2}\right)$ which is a homotopy between $B\left(F_{0}^{\prime}, F_{0}\right)=B\left(\psi_{2}, \psi_{1}\right)$ and $B\left(F_{1}^{\prime}, F_{1}\right)=$ $B\left(\psi_{2}^{\prime}, \psi_{1}^{\prime}\right)$.

The following technique is the main tool that we use to produce long exact sequences of homotopy groups.

Proposition 3.2 If $H$ is a normal closed subgroup of a topological group $G$, then there is a long exact sequence of homotopy groups:

$$
\cdots \rightarrow \pi_{n}(H) \rightarrow \pi_{n}(G) \rightarrow \pi_{n}(G / H) \rightarrow \pi_{n-1}(H) \rightarrow \cdots
$$

Proof From the fibrations
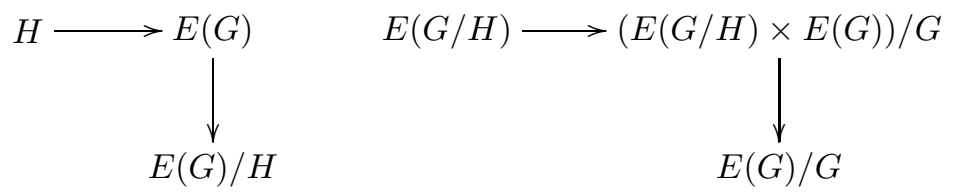
we see that $E(G) / H$ is a weak model of $B(H)$ and $(E(G / H) \times E(G)) / G$ is a weak model of $B(G)$. Since $(E(G / H) \times E(G)) / G=(E(G / H) \times(E(G) / H)) /(G / H)$, by the Borel construction, we have a fibration

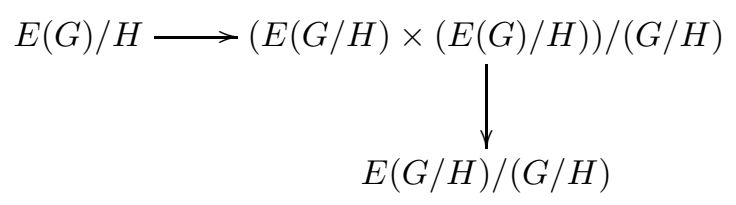

which induces the desire long exact sequence in homotopy groups.

\subsection{Relative Theory in Reduced Real Lawson Homology}

Definition Suppose that $X, Y$ are real projective varieties and $Y$ is a subvariety of $X$. Define the dimension $p$ relative reduced real cycle group to be

$$
R_{p}(X, Y)=\frac{R_{p}(X)}{R_{p}(Y)}
$$

Definition We say that a quasiprojective variety $U$ is real if there exist real projective varieties $X$ and $Y, Y \subset X$ such that $U=X-Y$. We define the $p$-th reduced real cycle group of $U$ to be $R_{p}(U)=R_{p}(X, Y)$.

We need to show that the definition of reduced real cycle groups of real quasiprojective varieties is independent of the choice of its compactification.

Lemma 3.3 Suppose that $X, Y$ are real projective varieties and $Y$ is a subvariety of $X$. Then $\frac{R_{r}(X)}{R_{r}(Y)}$ is isomorphic as a topological group to $\frac{Z_{r}(X, Y)_{\mathbb{R}}}{Z_{r}(X, Y)^{a v}}$.

Proof Let $Q_{1}, Q_{2}, q_{1}, q_{2}$ be the quotient maps and $\psi\left(x+Z_{r}(X)^{a v}+R_{r}(Y)\right)=$ $x+Z_{r}(Y)_{\mathbb{R}}+Z_{r}(X, Y)^{a v}$ as following:

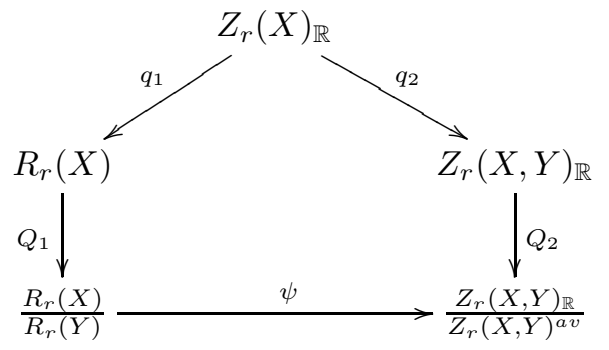

The diagram commutes and since those quotient maps are open maps, $\psi$ is continuous. Easy to check that $\psi$ is bijective. Since $\psi(U)=Q_{2} \circ q_{2} \circ q_{1}^{-1} \circ Q_{1}^{-1}(U)$ is open for an open set $U, \psi$ is an open map hence the inverse of $\psi$ is also continuous. 
Definition Suppose that $X, Y, X^{\prime}, Y^{\prime}$ are real projective varieties, and $Y \subset X$, $Y^{\prime} \subset X^{\prime}$. A regular map $f: X \rightarrow X^{\prime}$ is real if $f(\bar{x})=\overline{f(x)}$ for all $x \in X$. The pair $(X, Y)$ is said to be relatively isomorphic to the pair $\left(X^{\prime}, Y^{\prime}\right)$ if there is a real regular map $f: X \longrightarrow X^{\prime}$ such that $f$ induces an isomorphism as real quasiprojective varieties between $X-Y$ and $X^{\prime}-Y^{\prime}$, and we call $f$ a real relative isomorphism.

In [18, Theorem 4.3], Lima-Filho proved the following result.

Theorem 3.4 A relative isomorphism $f:\left(X, X^{\prime}\right) \rightarrow\left(Y, Y^{\prime}\right)$ induces an isomorphism of topological groups:

$$
f_{*}: \frac{Z_{r}(X)}{Z_{r}\left(X^{\prime}\right)} \rightarrow \frac{Z_{r}(Y)}{Z_{r}\left(Y^{\prime}\right)}
$$

for all $r \geq 0$.

Hence when we have a real relative isomorphism, its restriction to real cycle groups and averaged cycle groups gives us the following isomorphisms.

Proposition 3.5 Suppose that $f:(X, Y) \longrightarrow\left(X^{\prime}, Y^{\prime}\right)$ is a real relative isomorphism. Then $f$ induces a topological group isomorphism between

1. $Z_{r}(X, Y)_{\mathbb{R}}$ and $Z_{r}\left(X^{\prime}, Y^{\prime}\right)_{\mathbb{R}}$

2. $Z_{r}(X, Y)^{a v}$ and $Z_{r}\left(X^{\prime}, Y^{\prime}\right)^{a v}$

3. $\frac{Z_{r}(X, Y)_{\mathbb{R}}}{Z_{r}(X, Y)^{a v}}$ and $\frac{Z_{r}\left(X^{\prime}, Y^{\prime}\right)_{\mathbb{R}}}{Z_{r}\left(X^{\prime}, Y^{\prime}\right)^{a v}}$

Corollary 3.6 Suppose that $f:(X, Y) \longrightarrow\left(X^{\prime}, Y^{\prime}\right)$ is a real relative isomorphism, then $f$ induces a topological group isomorphism between $\frac{R_{r}(X)}{R_{r}(Y)}$ and $\frac{R_{r}\left(X^{\prime}\right)}{R_{r}\left(Y^{\prime}\right)}$.

Proof This follows directly from Lemma 3.3 and the proposition above.

Thus the reduced real cycle groups of a real quasiprojective variety are well defined. Now we are able to define the reduced real Lawson homology groups of a real quasiprojective variety.

Definition Let $X, Y$ be two real projective varieties and $Y$ be a subvariety of $X$. We define the relative reduced real Lawson homology groups of $(X, Y)$ to be

$$
R L_{p} H_{n}(X, Y)=\pi_{n-p}\left(R_{p}(X, Y)\right) .
$$

Suppose that $U$ is a real quasiprojective variety. Take a real compactification $(X, Y)$ of $U$. We define the reduced real Lawson homology groups of $U$ to be

$$
R L_{p} H_{n}(U)=\pi_{n-p}\left(R_{p}(X, Y)\right) .
$$


Proposition 3.7 Suppose that $X_{3} \subset X_{2} \subset X_{1}$ are real projective varieties. There is a long exact sequence in reduced real Lawson homology:

$\cdots R L_{p} H_{n}\left(X_{2}, X_{3}\right) \longrightarrow R L_{p} H_{n}\left(X_{1}, X_{3}\right) \longrightarrow R L_{p} H_{n}\left(X_{1}, X_{2}\right) \longrightarrow R L_{p} H_{n-1}\left(X_{2}, X_{3}\right) \longrightarrow \cdots$

Proof We have a short exact sequence of topological groups

$$
0 \rightarrow \frac{R_{p}\left(X_{2}\right)}{R_{p}\left(X_{3}\right)} \rightarrow \frac{R_{p}\left(X_{1}\right)}{R_{p}\left(X_{3}\right)} \rightarrow \frac{R_{p}\left(X_{1}\right)}{R_{p}\left(X_{2}\right)} \rightarrow 0
$$

and by Proposition 3.2 , we get the long exact sequence of homotopy groups.

Corollary 3.8 Suppose that $U, V$ are real projective varieties and $V \subset U$ is closed. Then there is a (localization) long exact sequence in reduced real Lawson homology:

$$
\cdots R L_{p} H_{n}(V) \longrightarrow R L_{p} H_{n}(U) \longrightarrow R L_{p} H_{n}(U-V) \longrightarrow R L_{p} H_{n-1}(V) \longrightarrow \cdots
$$

Proof Let $U=X_{1}-X_{3}$ where $X_{1}, X_{3}$ are real projective varieties and take the closure $\bar{V}$ of $V$ in $X_{1}$. Let $X_{2}=\bar{V} \cup X_{3}$. Then $X_{2}-X_{3}=V, X_{1}-X_{2}=U-V$ and $X_{3} \subset X_{2} \subset X_{1}$. By the Proposition above, we get the long exact sequence.

\subsection{Relative Theory in Reduced Real Morphic cohomol- ogy}

Definition Suppose that $X, Y$ are real projective varieties and the dimension of $Y$ is $n$. We define the codimension $t$ real cocycle group of $X$ with values in $Y$ to be

$$
R^{t}(Y)(X)=R_{n-t}(Y)(X),
$$

and the codimension $t$ reduced real cocycle group of $X$ to be

$$
R^{t}(X)=\frac{R_{0}\left(\mathbb{P}^{t}\right)(X)}{R_{0}\left(\mathbb{P}^{t-1}\right)(X)} .
$$

From Proposition 2.9. we know that $R_{0}\left(\mathbb{P}^{t-1}\right)(X) \hookrightarrow R_{0}\left(\mathbb{P}^{t}\right)(X)$ as a closed subgroup, thus $R^{t}(X)$ is a topological group.

To define relative reduced real morphic cohomology, we need to show that $R^{t}(X)$ acts on $R^{t}(Y)$ where $Y$ is a real subvariety of $X$.

Proposition 3.9 Suppose that $X, Y$ are real projective varieties and $Y$ is a subvariety of $X$. Then there is a topological group homomorphism

$$
\phi: R^{t}(X) \longrightarrow R^{t}(Y)
$$


Proof We have a commutative diagram:

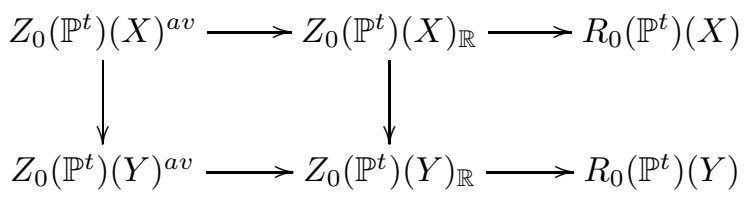

where maps in the horizontal direction are inclusions and maps in the vertical direction are restrictions. Hence they induce a map from $R_{0}\left(\mathbb{P}^{t}\right)(X)$ to $R_{0}\left(\mathbb{P}^{t}\right)(Y)$.

From the following commutative diagram:

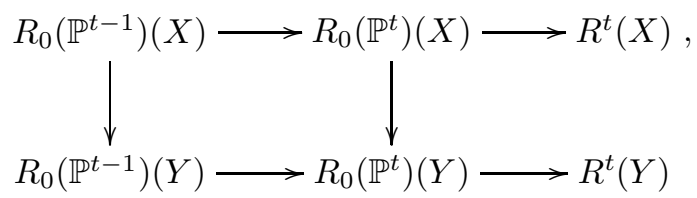

we see that there is a map from $R^{t}(X)$ to $R^{t}(Y)$.

Definition Suppose now $Y$ is a subvariety of $X$. Define the codimension $t$ relative reduced real cocycle group to be

$$
R^{t}(X \mid Y)=B\left(R^{t}(Y), R^{t}(X)\right)
$$

by the action from Proposition 3.9.

Definition The codimension $t$ reduced real morphic cohomology group of a real projective variety $X$ is defined to be

$$
R L^{t} H^{k}(X)=\pi_{t-k}\left(R^{t}(X)\right)
$$

Suppose now $X, Y$ are real projective varieties and $Y \subset X$. We define the relative reduced real morphic cohomology group to be

$$
R L^{t} H^{k}(X \mid Y)=\pi_{t-k}\left(R^{t}(X \mid Y)\right),
$$

and the reduced real bivariant $Y$-valued morphic cohomology to be

$$
R L^{t} H^{k}(X ; Y)=\pi_{t-k} R^{t}(Y)(X) .
$$

Theorem 3.10 (Long exact sequences) Long exact sequence in the reduced real morphic cohomology:

$\cdots \longrightarrow R L^{t} H^{k}(Y) \longrightarrow R L^{t} H^{k}(X \mid Y) \longrightarrow R L^{t} H^{k+1}(X) \longrightarrow R L^{t} H^{k+1}(Y) \longrightarrow \cdots$.

Proof This follows from the homotopy sequence induced by the Borel construction

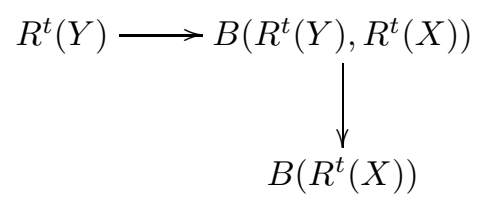


Remark It is much more difficult to generalize the reduced real morphic cohomology to real quasiprojective varieties. The generalization of morphic cohomology to quasiprojective varieties was done by Friedlander in [5]. It is quite possible that his approach may be adopted, but in this paper what we mainly concern are projective varieties, so we do not attempt to do that now.

\section{Functoriality}

There are analogous functorial properties between Lawson homology and reduced real Lawson homology, morphic cohomology and reduced real morphic cohomology. Similar results for bivariant morphic cohomology can be found in [6].

Proposition 4.1 Suppose that $X, X^{\prime}, Y, Y^{\prime}$ are real projective varieties and $f$ : $X^{\prime} \longrightarrow X, g: Y \longrightarrow Y^{\prime}$ are morphisms of real projective varieties.

(a) The "pullback of reduced real cocycles" determines a homomorphism

$$
\begin{aligned}
& f^{*}: R L^{s} H^{q}(X ; Y) \longrightarrow R L^{s} H^{q}\left(X^{\prime} ; Y\right), \\
& f^{*}: R L^{s} H^{q}(X) \rightarrow R L^{s} H^{q}\left(X^{\prime}\right) .
\end{aligned}
$$

(b) The "pushforward of reduced real cycles" determines a homomorphism

$$
\begin{gathered}
g_{*}: R L^{s} H^{q}(X ; Y) \longrightarrow R L^{s-c} H^{q-c}\left(X ; Y^{\prime}\right), \\
g_{*}: R L_{r} H_{p}(Y) \rightarrow R L_{r} H_{p}\left(Y^{\prime}\right)
\end{gathered}
$$

where $c=\operatorname{dim} Y-\operatorname{dim} Y^{\prime}$.

(c) Given morphisms $f_{2}: X^{\prime} \longrightarrow X, f_{1}: X^{\prime \prime} \longrightarrow X^{\prime}$ and $g_{1}: Y \longrightarrow Y^{\prime}$, $g_{2}: Y^{\prime} \longrightarrow Y^{\prime \prime}$ where $X, X^{\prime}, X^{\prime \prime}, Y, Y^{\prime}, Y^{\prime \prime}$ are all real projective varieties, then

$$
\left(f_{2} \circ f_{1}\right)^{*}=\left(f_{1}\right)^{*} \circ\left(f_{2}\right)^{*} \text { and }\left(g_{2} \circ g_{1}\right)_{*}=\left(g_{2}\right)_{*} \circ\left(g_{1}\right)_{*} .
$$

Proof (a) The pullback $f^{*}: Z^{s}(Y)(X) \longrightarrow Z^{s}(Y)\left(X^{\prime}\right)$ is a continuous map which is proved in [6. Proposition 2.4]. So we only need to verify that $f^{*}$ maps real cycles to real cycles and averaged cycles to averaged cycles. Since $f$ is real morphism, if $\alpha \in Z^{s}(Y)(X)_{\mathbb{R}}, f^{*} \alpha(\bar{x})=\alpha(f(\bar{x}))=\overline{\alpha(f(x))}$, hence $f^{*} \alpha \in Z^{s}(Y)\left(X^{\prime}\right)_{\mathbb{R}}$. For $\beta \in Z^{s}(Y)(X), f^{*}(\beta+\bar{\beta})=f^{*} \beta+\overline{f^{*} \beta} \in Z^{s}(Y)\left(X^{\prime}\right)^{a v}$. So it induces a map from $R^{s}(Y)(X)$ to $R^{s}(Y)\left(X^{\prime}\right)$, and therefore a group homomorphism in homotopy groups. From the commutative diagram

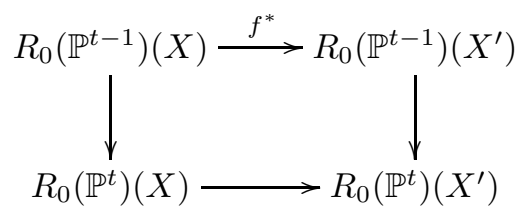


we see that $f^{*}$ induces a map $f^{*}: R^{t}(X) \rightarrow R^{t}\left(X^{\prime}\right)$.

(b) Let $n$ be the dimension of $Y$. The pushforward $g_{*}$ determines a continuous algebraic monoid homomorphism

$$
g_{*}: \mathscr{C}_{n-s}(Y) \rightarrow \mathscr{C}_{n-s}\left(Y^{\prime}\right),
$$

and by composition, a continuous homomorphism

$$
\mathcal{M o r}\left(X, \mathscr{C}_{n-s}(Y)\right) \rightarrow \operatorname{Mor}\left(X, \mathscr{C}_{n-s}\left(Y^{\prime}\right)\right)
$$

Passing to group completion we get a map $g_{*}: Z^{s}(Y)(X) \rightarrow Z^{s-c}\left(Y^{\prime}\right)(X)$. It is easy to see that $g_{*}$ maps real cycles to real cycles and averaged cycles to averaged cycles. Hence we get a map $g_{*}: R^{s}(Y)(X) \rightarrow R^{s-c}\left(Y^{\prime}\right)(X)$. When $X$ is a point, it reduces to the case $g_{*}: R_{r}(Y) \rightarrow R_{r}\left(Y^{\prime}\right)$.

(c) Since $\left(f_{2} \circ f_{1}\right)^{*}=f_{1}^{*} \circ f_{2}^{*}$ for $Y$-valued cocycle groups as proved in [6, Proposition 2.4], from (a), this relation passes to the quotient. Thus $\left(f_{2} \circ f_{1}\right)^{*}=$ $f_{1}^{*} \circ f_{2}^{*}$ in reduced $Y$-valued real cycle groups and reduced real cycle groups. A similar argument works for $g_{1}, g_{2}$.

Proposition 4.2 Let $f: X \longrightarrow X^{\prime}, g: Y^{\prime} \longrightarrow Y$ be morphisms of real projective varieties.

(a) If $f$ has equidimensional fibers, then there are Gysin homomorphisms

$$
\begin{gathered}
f_{!}: R L^{s} H^{q}(X ; Y) \longrightarrow R L^{s-c} H^{q-c}\left(X^{\prime} ; Y\right), \\
f_{!}: R L^{s} H^{q}(X) \rightarrow R L^{s-c} H^{q-c}\left(X^{\prime}\right)
\end{gathered}
$$

for all $s, q$ with $s \geq q \geq c$ where $c=\operatorname{dim}(X)-\operatorname{dim}\left(X^{\prime}\right)$.

(b) If $g$ is flat, then for all $s, q$ with $s \geq q$, there are Gysin homomorphisms

$$
\begin{gathered}
g^{!}: R L^{s} H^{q}(X ; Y) \longrightarrow R L^{s} H^{q}\left(X ; Y^{\prime}\right), \\
g^{!}: R L_{r} H_{p}(Y) \rightarrow R L_{r+e} H_{p+e}\left(Y^{\prime}\right)
\end{gathered}
$$

where $e=\operatorname{dim}\left(Y^{\prime}\right)-\operatorname{dim}(Y)$.

(c). If $f_{1}: X \longrightarrow X^{\prime}$ and $f_{2}: X^{\prime} \longrightarrow X^{\prime \prime}$ are as in part (a), or if $g_{2}: Y^{\prime} \longrightarrow$ $Y$ and $g_{1}: Y^{\prime \prime} \longrightarrow Y^{\prime}$ are as in part (b), then

$$
\left(f_{2} \circ f_{1}\right) !=\left(f_{2}\right) ! \circ\left(f_{1}\right) ! \text { and }\left(g_{2} \circ g_{1}\right)^{!}=\left(g_{1}\right)^{!} \circ\left(g_{2}\right)^{!} .
$$

Proof (a) Let $f$ ! be the composition of the following maps:

$Z_{n-s}(Y)(X) \rightarrow Z_{n+m-s}(X \times Y) \stackrel{(1 \times f)}{\longrightarrow} Z_{n-s+c}(X \times Y)\left(X^{\prime}\right) \stackrel{p_{*}}{\longrightarrow} Z_{n-s+c}(Y)\left(X^{\prime}\right)$

where $1 \times f: X \times Y \rightarrow X \times Y \times X^{\prime}$ is the map defined by sending $(x, y)$ to $(x, y, f(x))$ and $p: X \times Y \rightarrow Y$ is the projection. Then $f_{!}$is a real map and hence induces a map $f_{!}: R^{s}(Y)(X) \rightarrow R^{s-c}(Y)\left(X^{\prime}\right)$. By the Lawson 
suspension theorem 5.8 , we have a homotopy equivalence between $R_{c}\left(\mathbb{P}^{s}\right)(X)$ and $R_{0}\left(\mathbb{P}^{s-c}\right)(X)$. From the following commutative diagram

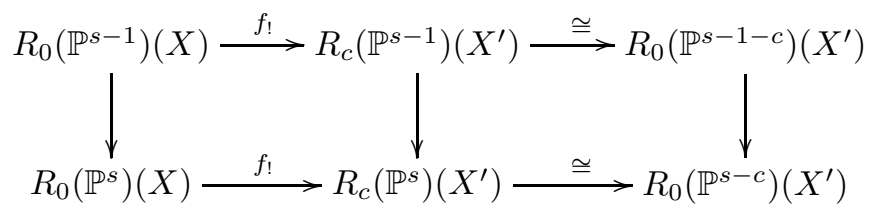

we see that $f_{!}$induces a map $f_{!}: R^{s}(X) \rightarrow R^{s-c}\left(X^{\prime}\right)$.

(b) Since $g$ is flat, we have a map $g^{!}: \mathscr{C}_{n-s}(Y) \rightarrow \mathscr{C}_{n-s+e}\left(Y^{\prime}\right)$ of pullback cycle. Then by composition, we get a map $g^{!}: \mathscr{M}$ or $\left(X, \mathscr{C}_{n-s}(Y)\right) \rightarrow$ $\mathscr{M} \operatorname{or}\left(X, \mathscr{C}_{n-s+e}\left(Y^{\prime}\right)\right)$ which induces a map after group completion $g^{!}: Z^{s}(Y)(X) \rightarrow$ $Z^{s}\left(Y^{\prime}\right)(X)$. It is obvious that $g^{!}$is real, hence it induces a map $g^{!}: R^{s}(Y)(X) \rightarrow$ $R^{s}\left(Y^{\prime}\right)(X)$. Take $X$ to be a point, we get $g^{!}: R_{r}(Y) \rightarrow R_{r+e}\left(Y^{\prime}\right)$. Taking homotopy groups, we complete the proof.

\section{Fundamental Properties}

\subsection{Homotopy Invariance}

Proposition 5.1 Let $X, X^{\prime}$ and $Y$ be real projective varieties. Suppose that $F: I \times X \longrightarrow X^{\prime}$ is a continuous map where $I$ is the unit interval and for each $t \in I, F_{t}$ is a real algebraic morphism. If $F_{0}(x)=f(x), F_{1}(x)=g(x)$ for all $x \in X$, then $f^{*}=g^{*}: R L^{*} H^{*}\left(X^{\prime} ; Y\right) \longrightarrow R L^{*} H^{*}(X ; Y)$ and they induce the same map $f^{*}=g^{*}: R L^{*} H^{*}\left(X^{\prime}\right) \longrightarrow R L^{*} H^{*}(X)$.

Proof Consider the map

$$
F^{*}: Z_{r}(Y)\left(X^{\prime}\right) \times \mathbb{C} \longrightarrow Z_{r}(Y)(X)
$$

which sends $(\sigma, t)$ to $F_{t}^{*} \sigma$. This map is continuous and is a homotopy between $f^{*}$ and $g^{*}$. Since each $F_{t}$ is a real morphism, by Proposition 4.1, $F_{t}^{*}$ passes to the quotient and thus $F$ induces a map $F^{*}: R_{r}(Y)\left(X^{\prime}\right) \times I \longrightarrow R_{r}(Y)(X)$ which is a homotopy between $F_{0}^{*}=f^{*}$ and $F_{1}^{*}=g^{*}$ so they induce the same map from $R L^{*} H^{*}\left(X^{\prime} ; Y\right)$ to $R L^{*} H^{*}(X ; Y)$. Furthermore, $F^{*}$ induces a homotopy $R_{0}\left(\mathbb{P}^{t-1}\right)\left(X^{\prime}\right) \times I \longrightarrow R_{0}\left(\mathbb{P}^{t-1}\right)(X)$ and a homotopy $R_{0}\left(\mathbb{P}^{t}\right)\left(X^{\prime}\right) \times I \longrightarrow$ $R_{0}\left(\mathbb{P}^{t}\right)(X)$ between the two maps induced by $f$ and $g$. Hence their induced maps from $R^{t}\left(X^{\prime}\right)$ to $R^{t}(X)$ are homotopic.

\subsection{The Splitting Principle}

Proposition 5.2 The real projective space $\mathbb{R P}^{d}$ is homeomorphic to $S P^{d}\left(\mathbb{P}^{1}\right)_{\mathbb{R}}$ where $S P^{d}\left(\mathbb{P}^{1}\right)_{\mathbb{R}}$ is the subset of $S P^{d}\left(\mathbb{P}^{1}\right)$ consisting of zero cycles invariant under the conjugation. 
Proof A proof of $\mathbb{P}^{d} \cong S P^{d}\left(\mathbb{P}^{1}\right)$ can be found in [12]. The main idea is sketched in the following. For $p=\left\{p_{1}, \ldots, p_{d}\right\} \in S P^{d}\left(\mathbb{P}^{1}\right)$, let $p_{i}=\left[-b_{i}: a_{i}\right] \in \mathbb{P}^{1}$ and we associate $p$ to a homogeneous polynomial

$$
P(x, y)=\prod_{i=1}^{d}\left(a_{i} x+b_{i} y\right)=\sum_{k=0}^{d} c_{k} x^{k} y^{d-k}
$$

where

$$
c_{k}=\sum_{|I|=k} a_{I} b_{I^{\prime}}
$$

and the sum is taken over all multi-indices $I=\left\{0 \leq i_{1}<\cdots<i_{k} \leq d\right\}$ of length $|I|=k$ and $I^{\prime}$ is the complementary multi-index with $\left|I^{\prime}\right|=d-k$. The map $\psi: S P^{d}\left(\mathbb{P}^{1}\right) \longrightarrow \mathbb{P}^{d}$ which maps $p$ to the point $\left[c_{0}: \cdots: c_{d}\right] \in \mathbb{P}^{d}$ is an isomorphism. The conjugation on $S P^{d}\left(\mathbb{P}^{1}\right)$ is given by the the conjugation of $\mathbb{P}^{d}$ through this isomorphism, i.e., $\bar{p}$ is defined to be $\psi^{-1} \overline{\psi(p)}$. Hence $\psi$ is real. This conjugation is same as the conjugation induced by the conjugation of $\mathbb{P}^{1}$ on $S P^{d}\left(\mathbb{P}^{1}\right)$, i.e., $\bar{p}=\left\{\overline{p_{1}}, \cdots, \overline{p_{d}}\right\}$. Since $\psi$ is a real homeomorphism, its restriction to $S P^{d}\left(\mathbb{P}^{1}\right)_{\mathbb{R}}$ gives a homeomorphism between $S P^{d}\left(\mathbb{P}^{1}\right)_{\mathbb{R}}$ and $\mathbb{R} \mathbb{P}^{d}$.

From section 2.10 of $[6]$, for $1 \leq t \leq s$, there is a monoid homomorphism

$$
S P\left(\mathbb{P}^{s}\right) \stackrel{p}{\longrightarrow} S P\left(\mathbb{P}^{t}\right)
$$

which is induced by the morphism

$$
p: \mathbb{P}^{s}=S P^{s}\left(\mathbb{P}^{1}\right) \longrightarrow S P^{\left(\begin{array}{c}
s \\
t
\end{array}\right)} S P^{t}\left(\mathbb{P}^{1}\right)
$$

defined by

$$
\left\{x_{1}, \ldots, x_{s}\right\} \longmapsto \sum_{|I|=t}\left\{x_{i_{1}}, \ldots, x_{i_{t}}\right\} .
$$

Let $\mathbb{P}^{0}=\left\{x_{0}\right\}$. We have an embedding $S P^{t}\left(\mathbb{P}^{1}\right) \hookrightarrow S P^{s}\left(\mathbb{P}^{1}\right)$ given by

$$
\left\{x_{1}, \ldots, x_{t}\right\} \mapsto\left\{x_{1}, \ldots, x_{t}, x_{0}, \ldots, x_{0}\right\}
$$

We extend this morphism additively to a monoid homomorphism

$$
p: S P\left(\mathbb{P}^{s}\right) \rightarrow S P\left(\mathbb{P}^{t}\right)
$$

and extend this morphism further to include the case $t=0$ by setting

$$
p\left(\sum n_{\alpha} x_{\alpha}\right)=\left(\sum n_{\alpha}\right) x_{0}
$$

for $x_{\alpha} \in \mathbb{P}^{s}$ and $n_{\alpha} \in \mathbb{Z}^{+}$. It is then easy to see that $p$ is real.

The map $p$ induces a homomorphism

$$
p: \mathscr{C}_{0}\left(\mathbb{P}^{s}\right)(X) \rightarrow \mathscr{C}_{0}\left(\mathbb{P}^{t}\right)(X)
$$


which passes to the group completion

$$
p: Z_{0}\left(\mathbb{P}^{s}\right)(X) \rightarrow Z_{0}\left(\mathbb{P}^{t}\right)(X)
$$

Since $p$ is real, the map also induces two maps

$$
p: Z_{0}\left(\mathbb{P}^{s}\right)(X)_{\mathbb{R}} \rightarrow Z_{0}\left(\mathbb{P}^{t}\right)(X)_{\mathbb{R}}
$$

and

$$
p: Z_{0}\left(\mathbb{P}^{s}\right)(X)^{a v} \rightarrow Z_{0}\left(\mathbb{P}^{t}\right)(X)^{a v}
$$

hence a map

$$
p^{\prime}: R_{0}\left(\mathbb{P}^{s}\right)(X) \rightarrow R_{0}\left(\mathbb{P}^{t}\right)(X)
$$

for $0 \leq t \leq s$.

The inclusion map $i: \mathbb{P}^{t} \longrightarrow \mathbb{P}^{s}$ induces an inclusion map $i^{\prime}: R_{0}\left(\mathbb{P}^{t}\right)(X) \longrightarrow$ $R_{0}\left(\mathbb{P}^{s}\right)(X)$ by Proposition 2.9 .

Lemma 5.3 The composition $\varphi^{\prime}=p^{\prime} \circ i^{\prime}: R_{0}\left(\mathbb{P}^{t}\right)(X) \longrightarrow R_{0}\left(\mathbb{P}^{t}\right)(X)$ is of the form

$$
\varphi^{\prime}=I d+\psi^{\prime}
$$

where image $\psi^{\prime} \subset R_{0}\left(\mathbb{P}^{t-1}\right)(X)$.

Proof Let $\varphi=p \circ i$ and $\psi=\varphi-I d$. Since $\varphi$ is real, $\psi$ is real. By [6, Lemma 2.11], $p \circ i(f)=(I d+\psi)(f)$ for $f \in Z_{0}\left(\mathbb{P}^{t}\right)(X)$ where the image of $\psi$ is contained in $Z_{0}\left(\mathbb{P}^{t-1}\right)(X)$. By the definition of $p^{\prime}$ and $i^{\prime}$, we have $p^{\prime} \circ i^{\prime}\left(f+Z_{0}\left(\mathbb{P}^{t}\right)(X)^{a v}\right)=$ $p \circ i(f)+Z_{0}\left(\mathbb{P}^{t}\right)(X)^{a v}=f+\psi(f)+Z_{0}\left(\mathbb{P}^{t}\right)(X)^{a v}$ for $f \in Z_{0}\left(\mathbb{P}^{t}\right)(X)_{\mathbb{R}}$. The map $\psi$ is real, thus $\psi\left(Z_{0}\left(\mathbb{P}^{t}\right)(X)_{\mathbb{R}}\right)$ is contained in $Z_{0}\left(\mathbb{P}^{t-1}\right)(X)_{\mathbb{R}}$. And it induces a $\operatorname{map} \psi^{\prime}: R_{0}\left(\mathbb{P}^{t}\right)(X) \rightarrow R_{0}\left(\mathbb{P}^{t}\right)(X)$ such that $p^{\prime} \circ i^{\prime}=\left(I d+\psi^{\prime}\right)$ and the image of $\psi^{\prime}$ is contained in $R_{0}\left(\mathbb{P}^{t-1}\right)(X)$.

Theorem 5.4 (Splitting Principle) For a real projective variety $X$, we have a homotopy equivalence:

$$
R_{0}\left(\mathbb{P}^{s}\right)(X) \cong R^{0}(X) \times R^{1}(X) \times \cdots \times R^{s}(X) .
$$

Proof Let $M^{t}=R_{0}\left(\mathbb{P}^{t}\right)(X), Q^{t}=\frac{M^{t}}{M^{t-1}}$. Let $p^{k, t}: M^{k} \rightarrow M^{t}$ be the map induced by $p$, and $q^{t}: M^{t} \rightarrow Q^{t}$ be the quotient map. Let $q^{k, t}: M^{k} \rightarrow Q^{t}$ be the map defined by $q^{k, t}=q^{t} \circ p^{k, t}$, and $\xi^{k, t}: M^{t} \hookrightarrow M^{k} \rightarrow Q^{0} \times \cdots Q^{t}$ defined by $\xi^{k}=\left(q^{k, 0} \circ i\right) \times \cdots \times\left(q^{k, t} \circ i\right)$. We use induction to show that the map

$$
M^{t} \stackrel{\xi^{k, t}}{\rightarrow} Q^{0} \times \cdots Q^{t}
$$

is a homotopy equivalence. When $t=0$, it is trivially true. We assume that it is true for $t-1$. We have a commutative diagram:

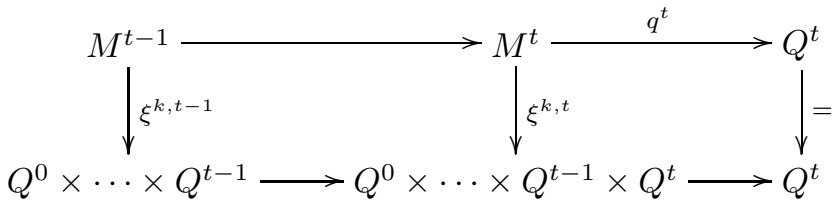


By Proposition 3.1, we see that $\xi^{k, t}$ induces an isomorphism of between homotopy groups of $M^{t}$ and $Q^{0} \times \cdots \times Q^{t}$. In Appendix, we show that $M^{t}$ and $Q^{0} \times \cdots \times Q^{t}$ have the homotopy type of a CW-complex, hence by Whitehead Theorem, $\xi^{k, t}$ is a homotopy equivalence.

\subsection{The Lawson Suspension Theorem}

Let $\mathbb{P}^{0}=\left\{x_{\infty}\right\}$. The suspension $\not X \subset \mathbb{P}^{n+1}$ of a projective variety $X$ is the complex cone over $X$, or equivalently, the Thom space of the hyperplane bundle $\mathcal{O}(1)$ of $\mathbb{P}^{n}$ restricted to $X$. A point in $\not X$ can be written as $[t: x]$ or $[1: 0: \ldots: 0]$ where $t \in \mathbb{C}, x \in X$. We consider $X$ as a subvariety of $\mathbb{Z} X$ by identifying $X$ with the zero section of $\not{\&} X$. If $X$ is a real projective variety, $\not{\&} X$ is also a real projective variety. The following Proposition is straight forward.

Proposition 5.5 1. Suppose that $\varphi: X \longrightarrow Y$ is a morphism of projective varieties where $X \subset \mathbb{P}^{n}, Y \subset \mathbb{P}^{m}$. Then the following diagram commutes:

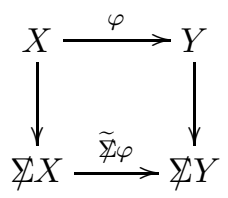

where $(\widetilde{\mathbb{Z}} \varphi)([x: t])=[\varphi(x): t]$ for all $t \in \mathbb{C}, x \in X$ and $(\widetilde{\mathbb{Z}} \varphi)([0: \ldots: 0:$ $1])=[0: \ldots: 0: 1]$ where the vertical arrows are inclusions.

2. If $\varphi: X \rightarrow Y$ is a morphism between real projective varieties, then $\widetilde{\mathbb{Z}} \varphi$ is real.

The above commutativity induces a commutative diagram of cycle groups.

Corollary 5.6 1. Suppose that $\varphi: X \longrightarrow Y$ is a morphism of projective varieties. Then the following diagram commutes:

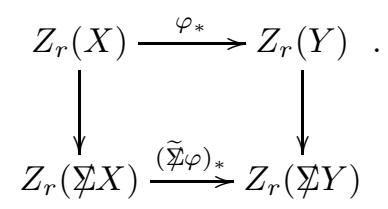

2. Suppose that $\varphi: X \longrightarrow Y$ is a morphism of real projective varieties. Then the following diagram commutes:

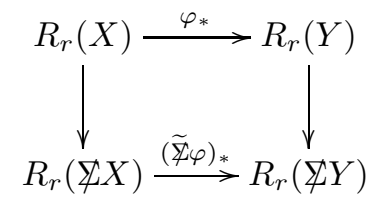


For $f \in \mathscr{M}$ or $\left(X, \mathscr{C}_{r}(Y)\right)$, define the suspension map $\mathbb{Z}_{*}: \mathscr{M}\left(X, \mathscr{C}_{r}(Y)\right) \longrightarrow$ $\mathscr{M}$ or $\left(X, \mathscr{C}_{r+1}(\not Y Y)\right)$ by $\left(\mathbb{Z}_{*} f\right)(x)=\not{\not} f(x)$ for $x \in X$, the point-wise suspension. Passing to the group completion, we get a map

$$
\mathbb{Z}_{*}: Z_{r}(Y)(X) \rightarrow Z_{r+1}(\not Y Y)(X)
$$

The Lawson suspension theorem for bivariant morphic cohomology is proved in [6. Theorem 3.3]. We state it in the following.

Theorem 5.7 For two projective varieties $X, Y$, the suspension map $Z_{*}: Z_{r}(Y)(X) \longrightarrow$ $Z_{r+1}(\not Y)(X)$ is a homotopy equivalence.

The Lawson suspension theorem for real cycle groups and averaged groups of projective spaces can be found in [17. The equivariant version of the Lawson suspension theorem can be found in [20. We generalize all previous results to bivariant reduced real morphic cohomology.

Suppose that $V$ is a projective variety. Two subvarieties of $V$ meet properly in $V$ if the codimension of each component of their intersection is the sum of the codimension in $V$ of that two subvarieties. When we say that a cycle $c_{1}$ meets another cycle $c_{2}$ properly we mean that each component of $c_{1}$ and $c_{2}$ meets properly.

Theorem 5.8 (Lawson suspension theorem) Suppose that $X, Y$ are real projective varieties and $Y \subset \mathbb{P}^{N}$. Then

1. $\mathbb{Z}_{*}: Z_{r}(Y)(X)_{\mathbb{R}} \longrightarrow Z_{r+1}(\not{Z} Y)(X)_{\mathbb{R}}$ is a homotopy equivalence.

2. 妆 $: Z_{r}(Y)(X)^{a v} \longrightarrow Z_{r+1}(\not Y Y)(X)^{a v}$ is a homotopy equivalence.

3. 䩣 $: R_{r}(Y)(X) \longrightarrow R_{r+1}(\mathbb{Z} Y)(X)$ is a homotopy equivalence.

\section{Proof}

Step 1:

Let

$T_{r+1}(\not Y Y)(X)=\left\{f \in Z_{r+1}(\not{Z} Y)(X) \mid f(x)\right.$ meets $x \times Y$ properly for all $\left.x \in X\right\}$,

$$
\begin{aligned}
T_{r+1}(\not{Z} Y)(X)_{\mathbb{R}} & =T_{r+1}(\not{Z} Y)(X) \cap Z_{r+1}(\not Y Y)(X)_{\mathbb{R}}, \\
T_{r+1}(\not{Z} Y)(X)^{a v} & =T_{r+1}(\not{Z} Y)(X) \cap Z_{r+1}(\not{Z} Y)(X)^{a v} .
\end{aligned}
$$

Following Proposition 3.2 in 4 , we define $\Lambda \subset \mathbb{P}^{N+1} \times \mathbb{P}^{1} \times \mathbb{P}^{N+1}$ to be the graph of the rational map $\mathbb{P}^{N+1} \times \mathbb{P}^{1} \longrightarrow \mathbb{P}^{N+1}$ whose restriction to $\mathbb{P}^{N+1} \times t$ for $t \in \mathbb{A}^{1}-\{0\}$ is the linear automorphism $\Theta_{t}: \mathbb{P}^{N+1} \longrightarrow \mathbb{P}^{N+1}$ sending $\left[z_{0}: \cdots: z_{N}: z_{N+1}\right]$ to $\left[z_{0}: \cdots: z_{N}: \frac{1}{t} z_{N+1}\right]$. More explicitly, $\Lambda$ is the closed subvariety given by the homogeneous equations:

$$
\left\{\begin{array}{l}
X_{i} Y_{j}-X_{j} Y_{i}=0 \\
T X_{N+1} Y_{j}-S X_{j} Y_{N+1}=0, \quad \text { for } 0 \leq i, j \leq N
\end{array}\right.
$$


where $\left[X_{0}: \cdots: X_{N+1}\right] \in \mathbb{P}^{N+1},[S: T] \in \mathbb{P}^{1}$, and $\left[Y_{0}: \cdots: Y_{N+1}\right] \in \mathbb{P}^{N+1}$.

For $f \in T_{r+1}(\not Y Y)(X), t \in \mathbb{R}-\{0\} \subset \mathbb{P}^{1}$, define

$$
\phi_{t}(f)=\operatorname{Pr}_{1,4 *}\left[(X \times \Lambda) \bullet\left(f \times t \times \mathbb{P}^{N+1}\right)\right]
$$

where $\operatorname{Pr}_{1,4}: X \times \mathbb{P}^{N+1} \times \mathbb{P}^{1} \times \mathbb{P}^{N+1} \longrightarrow X \times \mathbb{P}^{N+1}$ is the projection and it is proved in [4, Proposition 3.2], $\phi_{t}(f)(x)$ meets $x \times Y$ properly in $x \times \mathbb{Z Y}$ for all $x \in X$. For $t \in \mathbb{R}$, since $\phi_{t}$ is real, $\phi_{t}$ maps $T_{r+1}(\not{Z} Y)(X)_{\mathbb{R}}$ to $T_{r+1}(\not Z Y)(X)_{\mathbb{R}}$ and $T_{r+1}(\not Y Y)(X)^{a v}$ to $T_{r+1}(\not Y Y)(X)^{a v}$.

When $t=0$, check the equations defining $\Lambda$, we see that $\phi_{0}(f)=\mathbb{Z}(f \bullet(X \times$ $Y)) \in \mathbb{Z}_{*} Z_{r}(Y)(X)_{\mathbb{R}}$. When $t=1, \phi_{1}$ is the identity map. If $f \in \mathbb{Z}_{*} Z_{r}(Y)(X)$, $\phi_{t}(f)=f$ for $t \in \mathbb{R}$.

Therefore, $\phi_{0}: T_{r+1}(\not{Z} Y)(X)_{\mathbb{R}} \longrightarrow \mathbb{Z}_{*} Z_{r}(Y)(X)_{\mathbb{R}}$ and $\phi_{0}: T_{r+1}(\not{Z} Y)(X)^{a v} \longrightarrow$ $\mathbb{Z}_{*} Z_{r}(Y)(X)^{a v}$ are strong deformation retractions.

Step 2:

We use the notation $K_{r+1, e}(\not Y Y)(X)$ as in section 2. Let

$$
\begin{gathered}
T_{r+1, e}(\not \forall Y)(X)=K_{r+1, e}(\not Z Y)(X) \cap T_{r+1}(\not Z Y)(X), \\
T_{r+1, e}(\not Z Y)(X)_{\mathbb{R}}=T_{r+1}(\not \forall Y)(X)_{\mathbb{R}} \cap T_{r+1, e}(\not Z Y)(X),
\end{gathered}
$$

and

$$
T_{r+1, e}(\not Y)(X)^{a v}=T_{r+1}(\not Y Y)(X)^{a v} \cap T_{r+1, e}(\not Y Y)(X) .
$$

The second step is to show that the inclusion map $T_{r+1}(\mathbb{Z} Y)(X)_{\mathbb{R}} \hookrightarrow Z_{r+1}(\mathbb{Z} Y)(X)_{\mathbb{R}}$ and the inclusion map $T_{r+1}(\not Y)(X)^{a v} \hookrightarrow Z_{r+1}(\not Y Y)(X)^{a v}$ induce homotopy equivalences. We follow the proof of [6, Theorem 3.3]. Take two real points $t_{0}=[0: \cdots: 1], t_{1}=[0: \cdots: 1: 1] \in \mathbb{P}^{N+2}-\mathbb{P}^{N+1}$. Consider $\mathbb{P}^{N+2}$ as the algebraic suspension of $\mathbb{P}^{N+1}$ with respect to the point $t_{0}$ and let $p r$ : $\mathbb{P}^{N+2}-\left\{t_{1}\right\} \longrightarrow \mathbb{P}^{N+1}$ denote the linear projection away from $t_{1}$. Then consider the partially defined function

$$
\Psi_{e}: K_{r+1, d}(\not Y Y)(X) \times D i v_{e} \longrightarrow K_{r+1, d e}(\not Y Y)(X)
$$

given by

$$
\Psi_{e}(f, D)=(I d \times p r)((\not{Z} f) \bullet(X \times D))
$$

where Div $_{e}$ is the set of effective real divisors of degree $e$ on $\mathbb{P}^{N+2}$.

Let

$$
\triangle(f(x))=\left\{D \in \operatorname{Div}_{e}:(\not f(x) \bullet D) \text { does not meet }\{x\} \times H \text { properly }\right\}
$$

where $H \subset \mathbb{P}^{N+2}$ is the hyperplane containing $\mathbb{P}^{N} \cup\left\{t_{1}\right\}$ and $f \in K_{r+1, e}(\not Y Y)(X)$.

By [15, Lemma 5.11], one has

$$
\operatorname{codim}_{\mathbb{R}}(\triangle(f(x))) \geq\left(\begin{array}{c}
p+e+1 \\
e
\end{array}\right)
$$

where $p=\operatorname{dim} f(x)$. In particular, $\operatorname{codim}_{\mathbb{R}}(\triangle(f(x))) \longrightarrow \infty$ as $e \longrightarrow \infty$. 
We now choose $e(d)$ so that $\operatorname{codim}_{\mathbb{R}}(\triangle(f(x)))>\operatorname{dim} X+1$ for all $e>e(d)$. Then setting

$$
\triangle(f)=\cup_{x \in X} \triangle(f(x)),
$$

we have $\operatorname{codim}_{\mathbb{R}}(\triangle(f))>1$ for all $e>e(d)$. Consequently, for each $e>e(d)$, there must exist a line $L_{e} \subset$ Dive containing $e \cdot \mathbb{P}^{N+1}$ such that

$$
\left(L_{e}-e \cdot \mathbb{P}^{N+1}\right) \cap \triangle(f)=\emptyset .
$$

It follows immediately that $\Psi_{e}$ restricted to $K_{r+1, e}(\not Y)(X) \times\left(L_{e}-e \cdot \mathbb{P}^{N+1}\right)$ has images in $T_{r+1, d e}(\mathbb{Z} Y)(X)$ and this map is homotopic to the map given by multiplying by $e$. Since $\Psi_{e}$ is a real map, by restricting $\Psi_{e}$, we get two maps:

$$
\begin{gathered}
\Psi_{e}: K_{r+1, d}\left(\not{Z Y} Y(X)_{\mathbb{R}} \times\left(L_{e}-e \cdot \mathbb{P}^{N+1}\right) \longrightarrow T_{r+1, d e}(\not{Z} Y)(X)_{\mathbb{R}},\right. \\
\Psi_{e}: K_{r+1, d}(\not{Z} Y)(X)^{a v} \times\left(L_{e}-e \cdot \mathbb{P}^{N+1}\right) \longrightarrow T_{r+1, d e}(\not{Z} Y)(X)^{a v}
\end{gathered}
$$

which are both homotopic to the map given by multiplying by $e$. It then follows as in [15, Theorem 4.2] that the inclusions induce weak homotopy equivalences.

Consider the following commutative diagram:

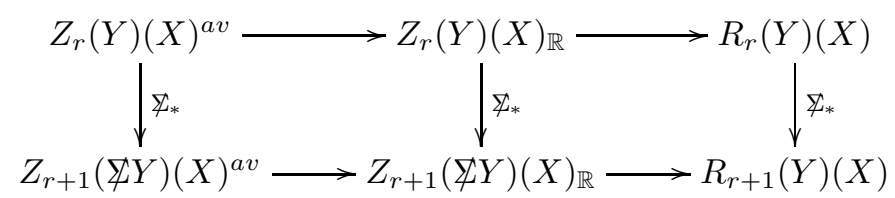

where the first two vertical arrows are homotopy equivalences induced by the suspension map. By Proposition 3.1 there is a weak homotopy equivalence induced by $\&$ from $R_{r}(Y)(X)$ to $R_{r+1}(\mathbb{Z} Y)(X)$. In Appendix we show that $Z_{r}(Y)(X)_{\mathbb{R}}, Z_{r}(Y)(X)^{a v}$, and $R_{r}(Y)(X)$ have the homotopy type of a CWcomplex. Hence by the Whitehead theorem, $\mathbb{Z}_{*}$ is a homotopy equivalence.

Take $X$ to be a real point, we have the Lawson Suspension Theorem for reduced real Lawson homology.

Corollary $5.9 R_{t}(X)$ is homotopy equivalent to $R_{t+1}(\not X)$ for $t \geq 0$.

Corollary $5.10 R_{t}\left(\mathbb{P}^{n}\right)$ is homotopy equivalent to $K\left(\mathbb{Z}_{2}, 0\right) \times K\left(\mathbb{Z}_{2}, 1\right) \times \cdots \times$ $K\left(\mathbb{Z}_{2}, n-t\right)$.

Proof By the Lawson suspension theorem 5.8, we have $R_{t}\left(\mathbb{P}^{n}\right) \cong R_{0}\left(\mathbb{P}^{n-t}\right)$. Since $R_{0}\left(\mathbb{P}^{n-t}\right)=\frac{Z_{0}\left(\mathbb{R}^{n-t}\right)}{2 Z_{0}\left(\mathbb{R}^{n-t}\right)}$ where $Z_{0}\left(\mathbb{R} \mathbb{P}^{n-t}\right)$ is the free abelian group generated by points of $\mathbb{R} \mathbb{P}^{n-t}$. By the Dold-Thom theorem, $\frac{Z_{0}\left(\mathbb{R}^{n-t}\right)}{2 Z_{0}\left(\mathbb{R}^{n-t}\right)}$ is homotopy equivalent to $K\left(\mathbb{Z}_{2}, 0\right) \times K\left(\mathbb{Z}_{2}, 1\right) \times \cdots \times K\left(\mathbb{Z}_{2}, n-t\right)$.

The following is the Lawson suspension theorem for reduced real cocycle groups. 
Corollary 5.11 $R^{t}(X)$ is homotopy equivalent to $\frac{R_{p}\left(\mathbb{P}^{p+t+1}\right)(X)}{R_{p}\left(\mathbb{P}^{p+t}\right)(X)}$.

Proof We have a commutative diagram where the vertical columns are homotopy equivalences from the suspension theorem:

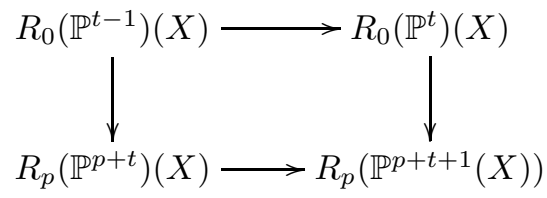

and then by Proposition 3.1, $R^{t}(X)$ is homotopy equivalent to $\frac{R_{p}\left(\mathbb{P}^{p+t+1}\right)}{R_{p}\left(\mathbb{P}^{p+t}(X)\right)}$.

Corollary 5.12 The group $R_{r}\left(\mathbb{C}^{n}\right)$ is homotopy equivalent to $K\left(\mathbb{Z}_{2}, n-r\right)$ for $r \leq n, n \in \mathbb{N}$.

Proof We have a commutative diagram:

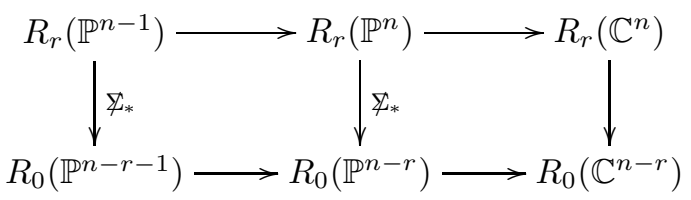

By Proposition 3.1 and the Dold-Thom theorem, we see that $R_{r}\left(\mathbb{C}^{n}\right)$ is homotopy equivalent to $K\left(\mathbb{Z}_{2}, n-r\right)$.

\subsection{Homotopy Property}

Theorem 5.13 Let $X$ be a real quasiprojective variety and $\pi: E \rightarrow X$ be a real algebraic vector bundle over $X$ of rank $k$, i.e., $E$ is a real quasiprojective variety and $\pi: E \rightarrow X$ is a complex algebraic vector bundle or rank $k$ with $\pi$ real. Then $\pi^{*}: R_{r}(X) \longrightarrow R_{r+k}(E)$ induces a homotopy equivalence.

Proof If the dimension of $X$ is 0 , then $E=\mathbb{C}^{k}$, this follows from Corollary 5.12 . Assume that the result is true for any real quasiprojective variety of dimension $\leq m$. Let the dimension of $X$ be $m+1$. Take a real quasiprojective variety $Y$ of dimension $\leq m$ which is closed in $X$ such that $E$ restricted to $U=X-Y$ is trivial. We have the following commutative diagram of topological groups:

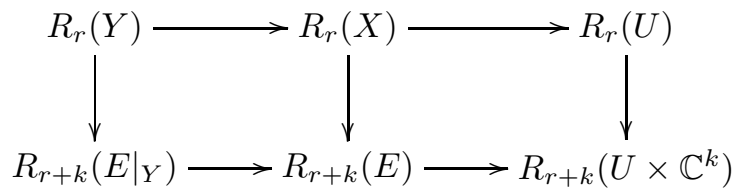

By the induction hypothesis, $\pi^{*}: R_{r}(Y) \longrightarrow R_{r+k}\left(\left.E\right|_{Y}\right)$ is a homotopy equivalence. Hence by Proposition 3.1, the result follows from the case of trivial 
bundles. By writing $\mathbb{C}^{k}=\mathbb{C}^{k-1} \times \mathbb{C}$, we see that it is sufficient to prove for the case of trivial line bundles.

Let $\bar{X}$ be a real projective closure of $X$. Take real projective varieties $Y, Y^{\prime} \subset$ $\bar{X}$ such that $X=\bar{X}-Y, U=\bar{X}-Y^{\prime}$ and the hyperplane line bundle $\left.\mathcal{O}_{\bar{X}}(1)\right|_{U}=$ $U \times \mathbb{C}$. We have the following commutative diagram of topological groups:

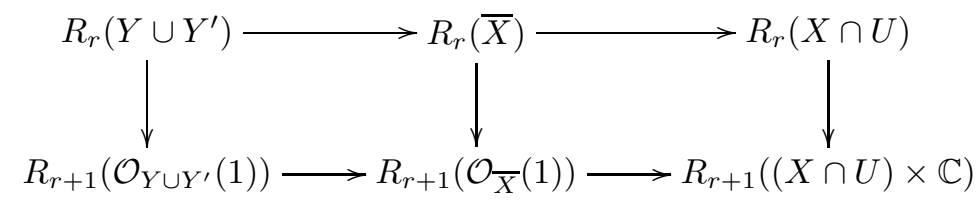

We note that for a real projective variety $W$,

$$
R_{r+1}\left(\mathcal{O}_{W}(1)\right)=\frac{R_{r+1}\left(\overline{\mathcal{O}_{\bar{W}}(1)}\right)}{R_{r+1}(\infty)}=R_{r+1}(\not W W) \cong R_{r}(W)
$$

Hence the first two vertical arrows are homotopy equivalences. It follows that

$$
\pi^{*}: R_{r}(X \cap U) \longrightarrow R_{r+1}((X \cap U) \times \mathbb{C})
$$

is a homotopy equivalence. Since $X=(X \cap U) \cup\left(X \cap Y^{\prime}\right)$, we have the following commutative diagram of topological groups:

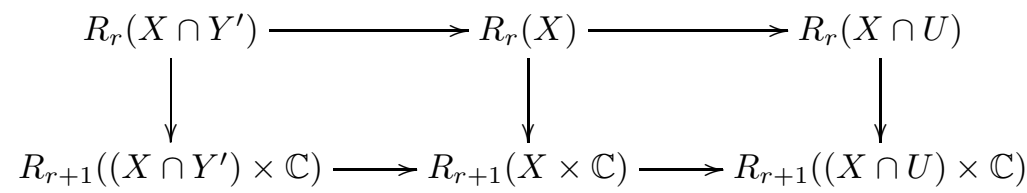

Since the dimension of $X \cap Y^{\prime}$ is less than the dimension of $X$, by induction on dimension, the left vertical arrow is a homotopy equivalence. Therefore, by Proposition 3.1, $\pi^{*}: R_{r}(X) \longrightarrow R_{r+1}(X \times \mathbb{C})$ is a homotopy equivalence.

\section{Duality Theorem}

The main ingredient of the proof of the duality theorem between morphic cohomology and Lawson homology is the moving lemma proved by Friedlander and Lawson in 7. Here we observe that their moving lemma preserves reality, and hence we have a moving lemma for real cycle groups and averaged cycle groups. Since we are not going to use the full power of the Friedlander-Lawson moving lemma, we state a simple form which fits our need.

Theorem 6.1 (Real Friedlander-Lawson Moving Lemma) Suppose that $X \subset$ $\mathbb{P}^{n}$ is a real projective variety of dimension $m$. Fix nonnegative integers $r, s, e$ where $r+s \geq m$. There exists a connected interval $I \subset \mathbb{R}$ containing 0 and a map

$$
\Psi: Z_{r}(X) \times I \longrightarrow Z_{r}(X)
$$

such that 
1. $\Psi_{0}=$ identity.

2. For $t \in I, \Psi_{t}$ is a real topological group homomorphism.

3. For all cycles $Y, Z$ of dimensions $r, s$ and degree $\leq e$ and all $t \neq 0$, any component of excess dimension(i.e., $>r+s-m)$ of $Y \bullet \Psi_{t}(Z)$ lies in the singular locus of $X$ for any $t \neq 0$.

Proof We give a brief explanation why their proof of the moving lemma can be reduced to real case. Let $F=\left(f_{0}, \cdots, f_{m}\right)$, a $(\mathrm{m}+1)$-tuples of real homogenous polynomials of degree $d$ in $\mathbb{P}^{n}$ where the zero locus of $F$ is disjoint from $X$. Via the Veronese embedding, we embed $\mathbb{P}^{n}$ into $\mathbb{P}^{K}$ where $K=\left(\begin{array}{c}n+d \\ d\end{array}\right)-1$. Let $\pi_{F}: \mathbb{P}^{M} \cdots \rightarrow \mathbb{P}^{m}$ be the linear projection determined by $F$ and $L(F)$ the center of $\pi_{F} . F$ determines a finite map $p_{F}: X \longrightarrow \mathbb{P}^{m}$, see for example [23]. For a cycle $Z, \pi_{F}^{*}(Z)=Z \# L(F), C_{F}(Z)=\pi_{F}^{*}\left(p_{F *}(Z)\right), R_{F}(Z)=C_{F}(Z) \bullet X-Z$ where \# is the join. Since $F$ is real, $\pi_{F}^{*}, C_{F}$ and $R_{F}$ are real maps. In [7, Proposition 2.3], a map

$$
\varphi_{L, D}(Z)=\pi_{0 *}\left((L \# Z) \bullet D_{1} \bullet \cdots \bullet D_{t}\right)
$$

is defined. We may take the effective divisors $D_{1}, \ldots, D_{t}$ to be real effective divisors, then $\varphi$ is a real map, furthermore, the proof and the conclusion remain the same.

Friedlander and Lawson construct the map $\Psi$ from the map

$\Psi_{\underline{N}}(Z, p)=(-1)^{m+1}(M+1) \cdot R_{F^{*}}(Z)+\sum_{i=1}^{m}(-1)^{i} \pi_{F^{i}}^{*}\left\{\Theta_{\underline{N}, p}\left\{p_{F^{i *}}\left(R_{F^{i-1}} \circ \cdots \circ R_{F^{0}}(Z)\right)\right\}\right\} \bullet X$

for some $\underline{N}$ large enough where $\underline{N}$ is a tuple of some positive integers, $M$ is a positive integer. The map $\Theta_{\underline{N}, p}$ is constructed by iterating the map $\varphi_{L, D}$ several times, so if we take $D$ to be a tuple of real effective divisors, $\Theta_{\underline{N}, p}$ is also a real map. It follows that $\Psi$ can be constructed to be a real map.

Now we are able to give a proof of a duality theorem between reduced real Lawson homology and reduced real morphic cohomology for nonsingular real projective varieties.

Theorem 6.2 Suppose that $X, Y$ are nonsingular real projective varieties and the dimension of $X$ is $m$. Then

1. $R_{r}(Y)(X)$ is homotopy equivalent to $R_{r+m}(X \times Y)$

2. $R^{t}(X)$ is homotopy equivalent to $R_{m}\left(X \times \mathbb{A}^{t}\right)$ for any $t \geq 0$

3. $R^{t}(X)$ is homotopy equivalent to $R_{m-t}(X)$ for $0 \leq t \leq m$.

Therefore, we have a group isomorphism

$$
R L^{t} H^{k}(X) \cong R L_{m-t} H_{m-k}(X)
$$

for $0 \leq t, k \leq m$. 
Proof For each $e \geq 0$, let

$$
K_{e}=\coprod_{d_{1}+d_{2} \leq e} \mathscr{C}_{r, d_{1}}(X \times Y) \times \mathscr{C}_{r, d_{2}}(X \times Y) / \sim
$$

and let

$$
K_{e}^{\prime}=\coprod_{d_{1}+d_{2} \leq e} \mathscr{C}_{r, d_{1}}(Y)(X) \times \mathscr{C}_{r, d_{2}}(Y)(X) / \sim
$$

where $\sim$ is the naive group completion relation and $\mathscr{C}_{r, d}(Y)(X)=\mathscr{C}_{r+m, d}(X \times$ $Y) \cap \mathscr{C}_{r}(Y)(X)$.

Let $q$ be the quotient map from $Z_{r+m}(X \times Y)_{\mathbb{R}}$ to $R_{r+m}(X \times Y)$ and let $q^{\prime}$ be the quotient map from $Z_{r}(Y)(X)_{\mathbb{R}}$ to $R_{r}(Y)(X)$. Let $\widetilde{K_{e}}=q\left(K_{e} \cap Z_{r+m}(X \times\right.$ $\left.Y)_{\mathbb{R}}\right), \widetilde{K_{e}^{\prime}}=q^{\prime}\left(K_{e}^{\prime} \cap Z_{r}(Y)(X)_{\mathbb{R}}\right)$.

By the real Friedlander-Lawson moving lemma, we get two real maps

$$
\begin{gathered}
\Psi: Z_{r+m}(X \times Y) \times I \longrightarrow Z_{r+m}(X \times Y), \\
\Psi^{\prime}: Z_{r}(Y)(X) \times I \longrightarrow Z_{r}(Y)(X)
\end{gathered}
$$

where $I$ is some connected interval containing 0 . By abuse of notation, we will use the same notation to denote the two maps induced by $\Psi, \Psi^{\prime}$ respectively

$$
\begin{gathered}
\Psi: R_{r+m}(X \times Y) \times I \longrightarrow R_{r+m}(X \times Y), \\
\Psi^{\prime}: R_{r}(Y)(X) \times I \longrightarrow R_{r}(Y)(X)
\end{gathered}
$$

Restricting $\Psi, \Psi^{\prime}$ to $\widetilde{K_{e}} \times I$ and $\widetilde{K_{e}^{\prime}} \times I$, we get two maps

$$
R \phi_{e}=\left.\widetilde{\Psi}\right|_{\widetilde{K}_{e} \times I}, \quad R \phi_{e}^{\prime}=\left.\widetilde{\Psi^{\prime}}\right|_{\widetilde{K}_{e}^{\prime} \times I} .
$$

The inclusion map $i: \mathscr{C}_{r}(Y)(X) \rightarrow \mathscr{C}_{r+m}(X \times Y)$ induces the FriedlanderLawson duality map

$$
\mathscr{D}: Z_{r}(Y)(X) \rightarrow Z_{r+m}(X \times Y)
$$

As shown in 8], this map is continuous, injective but is not a topological embedding.

Since $X, Y$ are real projective varieties, the duality map is real, hence it induces an injective map

$$
R \mathscr{D}: R_{r}(Y)(X) \longrightarrow R_{r+m}(X \times Y) .
$$

By Lemma 2.3, the filtrations

$$
\widetilde{K_{0}} \subset \widetilde{K_{1}} \subset \cdots=R_{r+m}(X \times Y)
$$

and

$$
\widetilde{K_{0}^{\prime}} \subset \widetilde{K_{1}^{\prime}} \subset \cdots=R_{r}(Y)(X)
$$

are locally compact and $R \mathscr{D}$ is filtration-preserving. 
We have the following commutative diagrams:
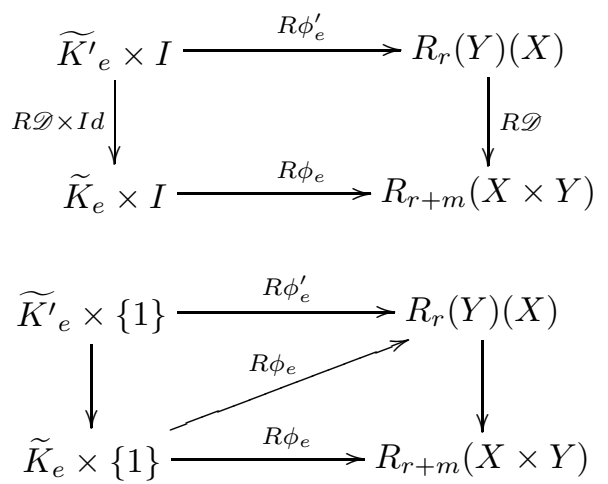

and there is a map $\widetilde{\lambda}_{e}=R \phi_{e}$ from $\widetilde{K}_{e} \times 1$ to $R_{r}(Y)(X)$. Thus by Lemma [5.2] in [8], $R_{r}(Y)(X)$ is homotopy equivalent to $R_{r+m}(X \times Y)$.

Furthermore, we have a commutative diagram of topological groups:

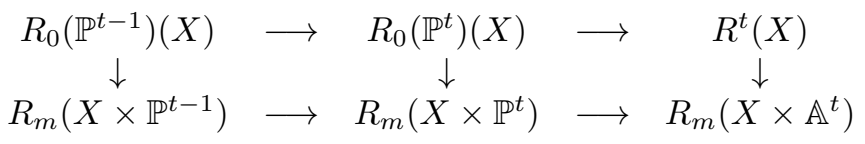

The first two vertical arrows are homotopy equivalences which implies the last one is also a homotopy equivalence. If $0 \leq t \leq m$, by the homotopy property of trivial bundle projection, $R^{t}(X)$ is homotopy equivalent to $R_{m-t}(X)$.

Proposition 6.3 Suppose that $X$ is a nonsingular real projective variety. Then $R_{0}\left(X \times \mathbb{A}^{t}\right)$ is homotopy equivalent to $\Omega^{-t} R_{0}(X)$ where $\Omega^{-t} R_{0}(X)$ is the $t$ fold delooping of $R_{0}(X)$ given the infinite loop space structure induced by the structure as a topological abelian group of $R_{0}(X)$.

Proof For two Eilenberg-Mac Lane spaces $K(G, i), K(H, j)$, denote

$$
K(G, i) \bigotimes K(H, j)=K(G \otimes H, i+j)
$$

and

$$
\left(\prod_{i=1}^{n}\left(K\left(G_{i}, i\right)\right)\right) \bigotimes\left(\prod_{j=1}^{m}\left(K\left(H_{j}, j\right)\right)\right)=\prod_{r=1}^{n+m} \prod_{i+j=r} K\left(G_{i} \otimes H_{j}, i+j\right) .
$$

From Theorem A.5. in [19, there is a canonical homotopy equivalence between $R_{0}(X)$ and $\prod_{k>0} K\left(H_{k}\left(R e X ; \mathbb{Z}_{2}\right), k\right)$. We will always consider the homotopic splitting of $\bar{R}_{0}(X)$ into Eilenberg-Mac Lane spaces by this canonical homotopy equivalence. By the Künneth formula for $\mathbb{Z}_{2}$-coefficients and the Dold-Thom theorem, we have $R_{0}\left(X \times \mathbb{P}^{t-1}\right)=R_{0}(X) \otimes R_{0}\left(\mathbb{P}^{t-1}\right)$ and $R_{0}\left(X \times \mathbb{P}^{t}\right)=R_{0}(X) \otimes R_{0}\left(\mathbb{P}^{t}\right)$. Since the inclusion map $\mathbb{P}^{t-1} \hookrightarrow \mathbb{P}^{t}$ induces 
an isomorphism $i_{*}: H_{k}\left(\mathbb{R P}^{t-1} ; \mathbb{Z}_{2}\right) \longrightarrow H_{k}\left(\mathbb{R P}^{t} ; \mathbb{Z}_{2}\right)$ for $0 \leq k<t$ in homology, $i_{*}: \pi_{k}\left(R_{0}\left(\mathbb{P}^{t-1}\right)\right) \longrightarrow \pi_{k}\left(R_{0}\left(\mathbb{P}^{t}\right)\right)$ is an isomorphism for $0 \leq k<t$ by the Dold-Thom theorem. Therefore $i_{*}: \pi_{l} R_{0}\left(X \times \mathbb{P}^{t-1}\right) \longrightarrow \pi_{l} R_{0}\left(X \times \mathbb{P}^{t}\right)$ is an isomorphism for $0 \leq l<t$ and an injection for $l \geq t$. From the long exact sequence

$$
\cdots \longrightarrow \pi_{l} R_{0}\left(X \times \mathbb{P}^{t-1}\right) \longrightarrow \pi_{l} R_{0}\left(X \times \mathbb{P}^{t}\right) \longrightarrow \pi_{l}\left(R_{0}\left(X \times \mathbb{A}^{t}\right)\right) \longrightarrow \cdots
$$

we see that $\pi_{l}\left(R_{0}\left(X \times \mathbb{A}^{t}\right)\right)=0$ if $0 \leq l<t$ and $\pi_{l}\left(R_{0}\left(X \times \mathbb{A}^{t}\right)\right)=\frac{\pi_{l} R_{0}\left(X \times \mathbb{P}^{t}\right)}{\pi_{l} R_{0}\left(X \times \mathbb{P}^{t-1}\right)}$ if $l \geq t$. Use the canonical homotopical splitting of $R_{0}(X)$ into a product of Eilenberg-Mac Lane spaces and denote the $i$-th component of the EilenbergMac Lane space of $R_{0}(X)$ to be $R_{0, i}(X)$. Then for $l \geq t$, from above calculation, we have $R_{0, l}\left(X \times \mathbb{A}^{t}\right)=R_{0, l-t}(X) \otimes R_{0, t}\left(\mathbb{P}^{t}\right)=R_{0, l-t}(X) \otimes K\left(\mathbb{Z}_{2}, t\right)=$ $\Omega^{-t} R_{0, l-t}(X)$. Thus $R_{0}\left(X \times \mathbb{A}^{t}\right)=\Omega^{-t} R_{0}(X)$.

By taking homotopy groups, we have the following result:

Corollary 6.4 For a nonsingular real projective variety $X$,

$$
R L_{0} H_{k}\left(X \times \mathbb{A}^{t}\right)= \begin{cases}R L_{0} H_{k-t}(X), & \text { if } k \geq t \\ 0, & \text { if } k<t .\end{cases}
$$

Corollary 6.5 Suppose that $X$ is a nonsingular real projective variety of dimension $m$ and its real points ReX is connected and of real dimension $m$. Then

$$
R L^{t} H^{k}(X)=R L^{m} H^{k}(X)=H^{k}\left(\operatorname{Re} X ; \mathbb{Z}_{2}\right)
$$

for $t \geq m \geq k$ and $R L^{t} H^{k}(X)=0$ for $t \geq k>m$.

Proof $R L^{t} H^{k}(X)=\pi_{t-k} R^{t}(X)=\pi_{t-k} R_{m}\left(X \times \mathbb{A}^{t}\right)=\pi_{t-k} R_{0}\left(X \times \mathbb{A}^{t-m}\right)=$ $\pi_{t-k} \Omega^{-(t-m)} R_{0}(X)$

$= \begin{cases}0, & \text { if } k>m \\ \pi_{m-k} R_{0}(X)=H_{m-k}\left(\operatorname{Re} X ; \mathbb{Z}_{2}\right)=H^{k}\left(\operatorname{Re} X ; \mathbb{Z}_{2}\right)=R L^{m} H^{k}(X) & \text { if } k \leq m\end{cases}$

Corollary 6.6 $R^{t}\left(\mathbb{P}^{n}\right)= \begin{cases}\prod_{i=0}^{t} K\left(\mathbb{Z}_{2}, i\right) & \text { if } t \leq n \\ \prod_{i=0}^{n} K\left(\mathbb{Z}_{2}, i+t-n\right) & \text { if } t>n\end{cases}$

Proof If $t \leq n$, by the Duality Theorem, $R^{t}\left(\mathbb{P}^{n}\right)=R_{n}\left(\mathbb{P}^{n} \times \mathbb{A}^{t}\right)=R_{n-t}\left(\mathbb{P}^{n}\right)=$ $\prod_{i=0}^{t} K\left(\mathbb{Z}_{2}, i\right) ;$ if $t>n$, then $R^{t}\left(\mathbb{P}^{n}\right)=R_{n}\left(\mathbb{P}^{n} \times \mathbb{A}^{t}\right)=R_{0}\left(\mathbb{P}^{n} \times \mathbb{A}^{t-n}\right)=$ $\Omega^{-(t-n)} R_{0}\left(\mathbb{P}^{n}\right)=\Omega^{-(t-n)} \prod_{i=0}^{n} K\left(\mathbb{Z}_{2}, i\right)=\prod_{i=0}^{n} K\left(\mathbb{Z}_{2}, i+t-n\right)$.

\section{Operations and Maps}

\subsection{Natural Transformations From Reduced Real Mor- phic Cohomology To Singular Cohomology}

Suppose that $X, Y$ are real projective varieties. It is a general fact that for a topological group $G$ and a locally compact Hausdorff space $A, \operatorname{Map}(A, G)$, 
the space of all continuous functions from $A$ to $G$ with the compact-open topology, is a topological group under point-wise multiplication. By using the graphing construction of Friedlander and Lawson, there is a natural transformation $j: Z_{r}(Y)(X) \rightarrow \operatorname{Map}\left(X, Z_{r}(Y)\right)$ induced by the inclusion map $i: \mathscr{M}$ or $\left(X, \mathscr{C}_{r}(Y)\right) \rightarrow \operatorname{Map}\left(X, Z_{r}(Y)\right)$. The restriction of $j$ to real cocycles gives us a map $j: Z_{r}(Y)(X)_{\mathbb{R}} \rightarrow \operatorname{Map}\left(X, Z_{r}(Y)\right)$. For $f \in Z_{r}(Y)(X)_{\mathbb{R}}$, if we restrict $f$ to $\operatorname{Re}(X)$, the set of real points of $X$, since $f(x)=\overline{f(\bar{x})}=\overline{f(x)}$, the image of $f$ lies in $Z_{r}(Y)_{\mathbb{R}}$. Composing the map $j$ with the restriction map, we have a continuous map

$$
\Psi^{\prime}: Z_{r}(Y)(X)_{\mathbb{R}} \longrightarrow M a p\left(\operatorname{Re} X, Z_{r}(Y)_{\mathbb{R}}\right)
$$

Composing again with the quotient map $q: Z_{r}(Y)_{\mathbb{R}} \longrightarrow R_{r}(Y)$, we have a continuous map

$$
\Psi^{\prime \prime}: Z_{r}(Y)(X)_{\mathbb{R}} \longrightarrow M a p\left(\operatorname{Re} X, R_{r}(Y)\right) .
$$

If $f=g+\bar{g}, \Psi^{\prime \prime}(f)(x)=g(x)+\overline{g(\bar{x})}+Z_{r}(Y)^{a v}=g(x)+\overline{g(x)}+Z_{r}(Y)^{a v}=$ $Z_{r}(Y)^{a v}$, so $Z_{r}(Y)(X)^{a v} \subset \operatorname{Ker} \Psi^{\prime \prime}$, therefore $\Psi^{\prime \prime}$ induces a continuous map

$$
\Psi: R_{r}(Y)(X) \longrightarrow M a p\left(\operatorname{Re} X, R_{r}(Y)\right)
$$

We summarize the above construction in the following diagram:

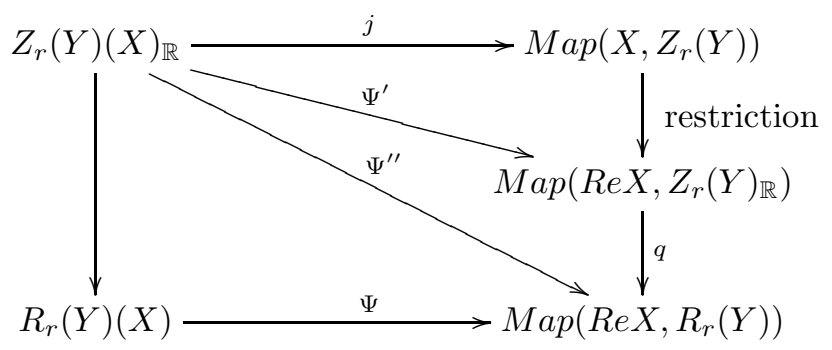

Now we are going to construct a map from $R^{t}(X)$ to $\operatorname{Map}\left(\operatorname{Re} X, R_{0}\left(\mathbb{A}^{t}\right)\right)$.

Let $q^{\prime}: R_{0}\left(\mathbb{P}^{t}\right) \longrightarrow R_{0}\left(\mathbb{A}^{t}\right)$ be the quotient map. Then $q^{\prime}$ induces a map, also denoted as $q^{\prime}$, from $\operatorname{Map}\left(\operatorname{Re} X, R_{0}\left(\mathbb{P}^{t}\right)\right)$ to $\operatorname{Map}\left(\operatorname{Re} X, R_{0}\left(\mathbb{A}^{t}\right)\right)$. Let $\Phi=$ $q^{\prime} \circ \Psi: R_{0}\left(\mathbb{P}^{t}\right)(X) \longrightarrow \operatorname{Map}\left(\operatorname{Re} X, R_{0}\left(\mathbb{A}^{t}\right)\right)$. For $f \in R_{0}\left(\mathbb{P}^{t-1}\right)(X), f \in \operatorname{ker}(\Phi)$, so $\Phi$ induces a map $\Phi^{t}: R^{t}(X) \longrightarrow \operatorname{Map}\left(\operatorname{Re} X, R_{0}\left(\mathbb{A}^{t}\right)\right)$. We summarize the construction of $\Phi^{t}$ in following:

$$
\Phi^{t}\left(f+Z_{0}\left(\mathbb{P}^{t}\right)(X)^{a v}+R_{0}\left(\mathbb{P}^{t-1}\right)(X)\right)=q^{\prime} \circ q \circ\left(\left.f\right|_{R e X}\right) .
$$

Since $R_{0}\left(\mathbb{A}^{t}\right)$ is the Eilenberg-Mac Lane space $K\left(\mathbb{Z}_{2}, t\right)$, taking homotopy groups, $\Phi^{t}$ induces a group homomorphism:

$$
\Phi^{t, k}: R L^{t} H^{k}(X) \longrightarrow H^{k}\left(\operatorname{Re} X ; \mathbb{Z}_{2}\right)
$$

Proposition 7.1 $\Phi^{t, k}: R L^{t} H^{k}(X) \longrightarrow H^{k}\left(R e X ; \mathbb{Z}_{2}\right)$ is a natural transformation for each $k$ with $0 \leq k \leq t$. 
Proof Suppose that $f: X \longrightarrow Y$ is a morphism between two real projective varieties. We have a pullback $f^{*}: R^{t}(Y) \longrightarrow R^{t}(X)$ (see Proposition 4.1) by mapping $\phi+R_{0}\left(\mathbb{P}^{t-1}\right)(Y)$ to $\phi \circ f+R_{0}\left(\mathbb{P}^{t-1}\right)(X)$. Since $f$ is a real map, it maps $\operatorname{Re}(X)$ to $\operatorname{Re}(Y)$, thus $f$ induces a map $f^{*}: \operatorname{Map}\left(\operatorname{Re} Y, R_{0}\left(\mathbb{A}^{t}\right)\right) \longrightarrow$ $\operatorname{Map}\left(\operatorname{Re} X, R_{0}\left(\mathbb{A}^{t}\right)\right)$. It is easy to check that the following diagram commutes:

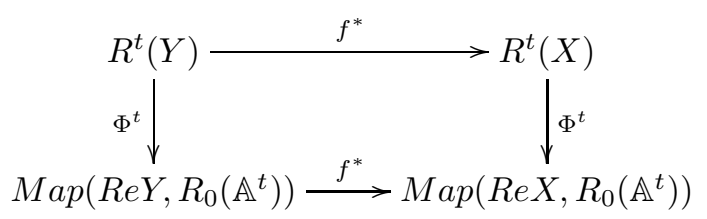

which then induces a commutative diagram of homotopy groups. For $g: Y \rightarrow$ $Z$ another morphism between real projective varieties, we have $\Phi^{t, k}(g \circ f)=$ $\Phi^{t, k}(f) \circ \Phi^{t, k}(g)$.

\subsection{Cup Product}

Suppose that $X, Y$ are two real projective varieties. The join $X \# Y$ of $X, Y$ is again real. If $\left[x_{0}: \ldots: x_{n}: y_{0}: \ldots: y_{m}\right] \in X \# Y$, the conjugation on $X \# Y$ is defined by $\overline{\left[x_{0}: \ldots: x_{n}: y_{0}: \ldots: y_{m}\right]}=\left[\bar{x}_{0}: \ldots: \bar{x}_{n}: \bar{y}_{0}: \ldots: \bar{y}_{m}\right]$.

From Section 6 in [6], there is a continuous pairing induced by the join of varieties:

$$
Z^{s}(Y)(X) \wedge Z^{s^{\prime}}\left(Y^{\prime}\right)\left(X^{\prime}\right) \stackrel{\#}{\longrightarrow} Z^{s+s^{\prime}}\left(Y \# Y^{\prime}\right)\left(X \times X^{\prime}\right)
$$

given by

$$
\left(\varphi \# \varphi^{\prime}\right)\left(x, x^{\prime}\right)=(\varphi(x)) \#\left(\varphi^{\prime}\left(x^{\prime}\right)\right)
$$

It is easy to see that this pairing is real. Therefore, for real projective varieties $X, X^{\prime}, Y, Y^{\prime}$, \# reduces to a continuous pairing

$$
R^{s}(Y)(X) \wedge R^{s^{\prime}}\left(Y^{\prime}\right)\left(X^{\prime}\right) \stackrel{\#}{\longrightarrow} R^{s+s^{\prime}}\left(Y \# Y^{\prime}\right)\left(X \times X^{\prime}\right) .
$$

Taking homotopy groups, we have a pairing:

$$
R L^{s} H^{q}(X ; Y) \otimes R L^{s^{\prime}} H^{q^{\prime}}\left(X^{\prime} ; Y^{\prime}\right) \longrightarrow R L^{s+s^{\prime}} H^{q+q^{\prime}}\left(X \times X^{\prime} ; Y \# Y^{\prime}\right),
$$

and when restricted to the diagonal of $X \times X$, it determines a cup product:

$$
R L^{s} H^{q}(X ; Y) \otimes R L^{s^{\prime}} H^{q^{\prime}}\left(X ; Y^{\prime}\right) \stackrel{\#}{\longrightarrow} R L^{s+s^{\prime}} H^{q+q^{\prime}}\left(X ; Y \# Y^{\prime}\right) .
$$

Now take $Y=\mathbb{P}^{s}, Y^{\prime}=\mathbb{P}^{s^{\prime}}$, the get a pairing

$$
R_{0}\left(\mathbb{P}^{s}\right)(X) \wedge R_{0}\left(\mathbb{P}^{s^{\prime}}\right)(X) \longrightarrow R_{1}\left(\mathbb{P}^{s+s^{\prime}+1}\right)(X \times X)
$$

Since

$$
R_{0}\left(\mathbb{P}^{s-1}\right)(X) \wedge R_{0}\left(\mathbb{P}^{s^{\prime}}\right)(X) \rightarrow R_{1}\left(\mathbb{P}^{s+s^{\prime}}\right)(X)
$$


and

$$
R_{0}\left(\mathbb{P}^{s}\right)(X) \wedge R_{0}\left(\mathbb{P}^{s^{\prime}-1}\right)(X) \rightarrow R_{1}\left(\mathbb{P}^{s+s^{\prime}}\right)(X),
$$

the pairing reduces to a pairing on the reduced real cocycle groups :

$$
R^{s}(X) \wedge R^{s^{\prime}}(X) \longrightarrow \frac{R_{1}\left(\mathbb{P}^{s+s^{\prime}+1}\right)(X)}{R_{1}\left(\mathbb{P}^{s+s^{\prime}}\right)(X)} \stackrel{\mathbb{\Psi}^{-1}}{\longrightarrow} R^{s+s^{\prime}}(X \times X)
$$

By restricting to the diagonal of $X \times X$, we get a pairing

$$
R^{s}(X) \wedge R^{s^{\prime}}(X) \longrightarrow R^{s+s^{\prime}}(X)
$$

which gives us a commutative cup product in reduced real morphic cohomology:

$$
R L^{s} H^{q}(X) \otimes R L^{s^{\prime}} H^{q^{\prime}}(X) \longrightarrow R L^{s+s^{\prime}} H^{q+q^{\prime}}(X) .
$$

Proposition 7.2 Suppose that $X, X^{\prime}, Y, Y^{\prime}, W, W^{\prime}, Z, Z^{\prime}$ are all real projective varieties and $f: X \longrightarrow X^{\prime}, g: W \longrightarrow W^{\prime}$ are real morphisms. Then we have the following commutative diagrams:

1.

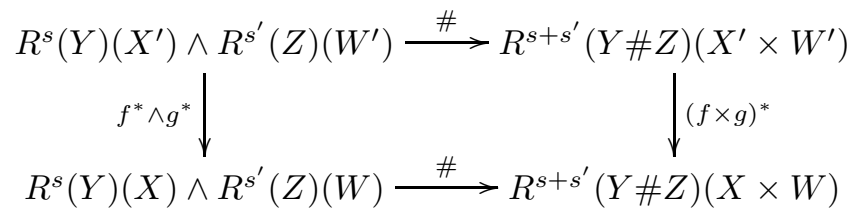

2.

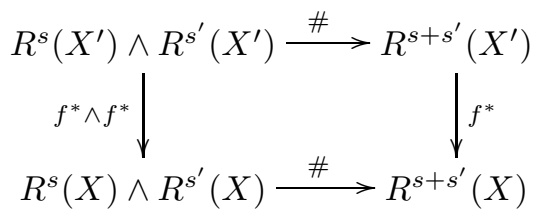

3.

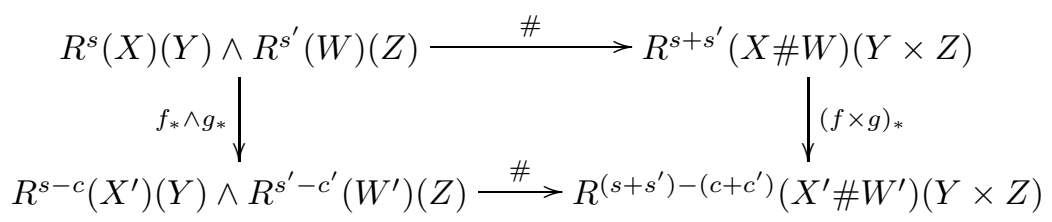

where $c=\operatorname{dim} X-\operatorname{dim} X^{\prime}, c^{\prime}=\operatorname{dim} W-\operatorname{dim} W^{\prime}$.

In short, cup product in reduced real morphic cohomology is natural with respect to real morphisms.

Proof We observe that

$$
\begin{gathered}
\left(f^{*} \varphi\right) \#\left(g^{*} \varphi^{\prime}\right)=(\varphi \circ f) \#\left(\varphi^{\prime} \circ g\right)=\left(\varphi \# \varphi^{\prime}\right) \circ(f \times g)=(f \times g)^{*}\left(\varphi \# \varphi^{\prime}\right), \\
\left(f_{*} \alpha\right) \#\left(g_{*} \alpha^{\prime}\right)=(f \times g)_{*}\left(\alpha \# \alpha^{\prime}\right)
\end{gathered}
$$

and then check that they pass to reduced real cocycles. 
From Lawson suspension theorem, we obtain a canonical homotopy equivalence:

$$
Z^{q}\left(\mathbb{P}^{n}\right) \longrightarrow \prod^{q} \stackrel{\text { def }}{=} \prod_{k=0}^{q} K(\mathbb{Z}, 2 k)
$$

for all $n \geq q$. In [21, Lawson and Michelsohn showed that the complex join

$$
\#_{\mathbb{C}}: \prod^{q} \times \prod^{q^{\prime}} \longrightarrow \prod^{q+q^{\prime}}
$$

has the property that

$$
\#_{\mathbb{C}}^{*}\left(\iota_{2 k}\right)=\sum_{r+s=k} \iota_{2 r} \otimes \iota_{2 s}
$$

in integral cohomology where $\iota_{2 k}$ is the generator of

$$
H^{2 k}(K(\mathbb{Z}, 2 k) ; \mathbb{Z}) \cong \mathbb{Z} .
$$

The cup product and cross product in cohomology are characterized by some axioms which can be found for example in [1]. From the above result, it is not difficult to check that the complex join induces the cross product in integral cohomology. Following a similar approach, Lam in [17] showed that the corresponding result holds for the pairing:

$$
\#_{\mathbb{R}}: \prod_{\mathbb{R}}^{q} \times \prod_{\mathbb{R}}^{q^{\prime}} \longrightarrow \prod_{\mathbb{R}}^{q+q^{\prime}}
$$

where $\prod_{\mathbb{R}}^{q}=K\left(\mathbb{Z}_{2}, 0\right) \times K\left(\mathbb{Z}_{2}, 1\right) \times \cdots \times K\left(\mathbb{Z}_{2}, q\right)$, thus \# induces the cross product in $\mathbb{Z}_{2}$-cohomology.

We now show that the natural transformation from reduced real morphic cohomology to $\mathbb{Z}_{2}$-singular cohomology is a ring homomorphism.

Proposition 7.3 Suppose that $X$ and $X^{\prime}$ are real projective varieties. Then for all $s, s^{\prime}$ and $q, q^{\prime}$, there are commutative diagrams:

1.

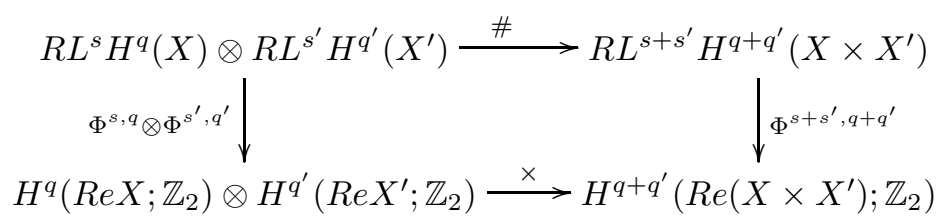

where the lower horizontal arrow is the usual cross product in $\mathbb{Z}_{2}$-coefficients.

2.

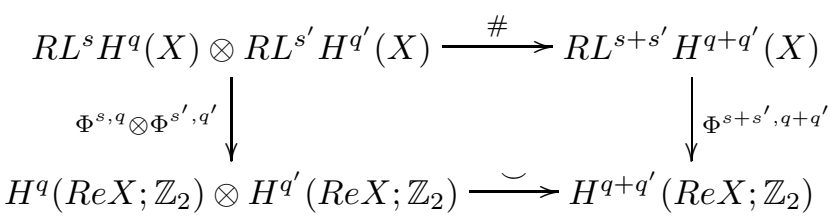

where $\smile$ is the cup product in singular cohomology with $\mathbb{Z}_{2}$-coefficients. 
In particular, the map

$$
\Phi^{*, *}: R L^{*} H^{*}(X) \longrightarrow H^{*}\left(\operatorname{Re} X ; \mathbb{Z}_{2}\right)
$$

is a graded-ring homomorphism.

Proof Consider the following commutative diagram:

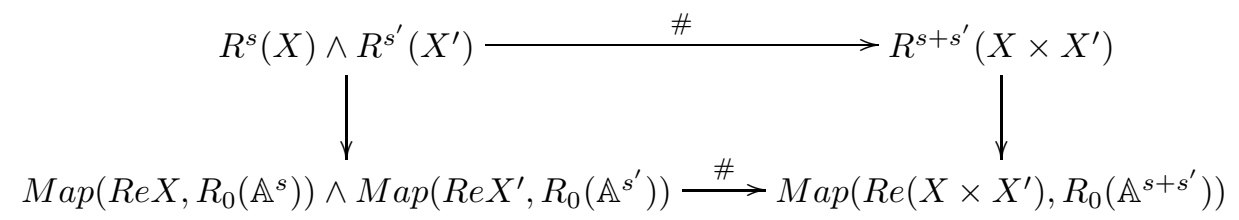

Since the lower arrow in the diagram above is exactly the map classifying the cross product in $\mathbb{Z}_{2}$-cohomology, taking homotopy groups, proves the first assertion. Let $X^{\prime}=X$ and $\Delta: X \longrightarrow X \times X$ be the diagonal map. Since $x \smile y=\Delta^{*}(x \times y)$ for $x, y \in H^{q}\left(\operatorname{Re} X ; \mathbb{Z}_{2}\right)$, we complete the proof by composing the first diagram with the following commutative diagram:

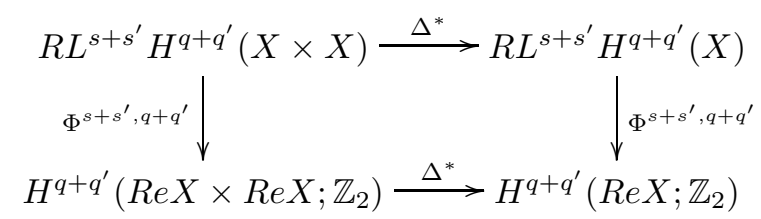

\subsection{The $S$-Map In Reduced Real Morphic Cohomology}

Let us give a construction of the $S$-map in reduced real morphic cohomology parallel to the construction of Lima-Filho in Lawson homology. Fix $x_{\infty} \in \mathbb{R} \mathbb{P}^{1}$ and consider the following diagram

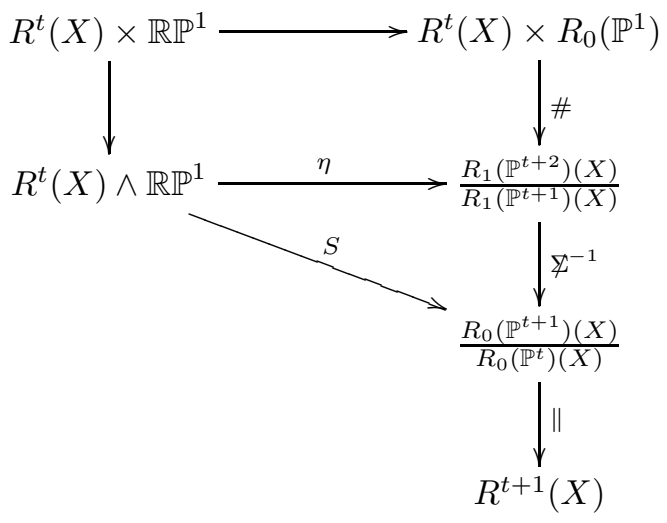

The top horizontal row sends $\left(f+R_{0}\left(\mathbb{P}^{t-1}\right)(X), x\right) \in R^{t}(X) \times \mathbb{R} \mathbb{P}^{1}$ to $(f+$ $\left.R_{0}\left(\mathbb{P}^{t-1}\right)(X), x-x_{\infty}+Z_{0}\left(\mathbb{P}^{1}\right)^{a v}\right)$ and the join on the right hand side sends 
$\left(f+R_{0}\left(\mathbb{P}^{t-1}\right)(X), y\right)$ to $f \# y+R_{1}\left(\mathbb{P}^{t+1}\right)(X)$ where $f \# y \in R_{1}\left(\mathbb{P}^{t+2}\right)(X)$. This map reduces to map $\eta$ from $R^{t}(X) \wedge \mathbb{R P}^{1}$ to $\frac{R_{1}\left(\mathbb{P}^{t+2}\right)(X)}{R_{1}\left(\mathbb{P}^{t+1}\right)(X)}$. Define the $S$-map to be $\mathbb{Z}^{-1} \circ \eta$ where $\mathbb{Z}^{-1}$ is the homotopy inverse of the suspension map.

So we have a map from $R^{t}(X) \wedge \mathbb{S}^{1} \longrightarrow R^{t+1}(X)$ which induces a map in the reduced real morphic cohomology:

$$
S: R L^{t} H^{k}(X) \longrightarrow R L^{t+1} H^{k}(X) .
$$

Proposition 7.4 For a real projective variety $X$, the following diagram commutes:

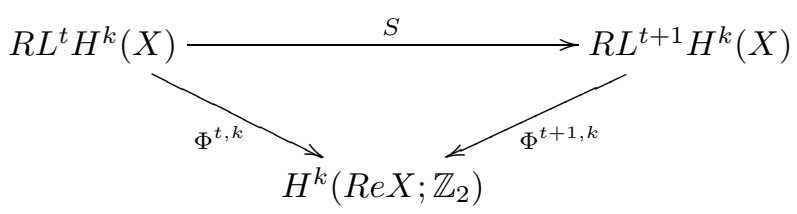

Proof Fix a point $x_{\infty} \in \mathbb{R P}^{1}$. Consider the following diagram:

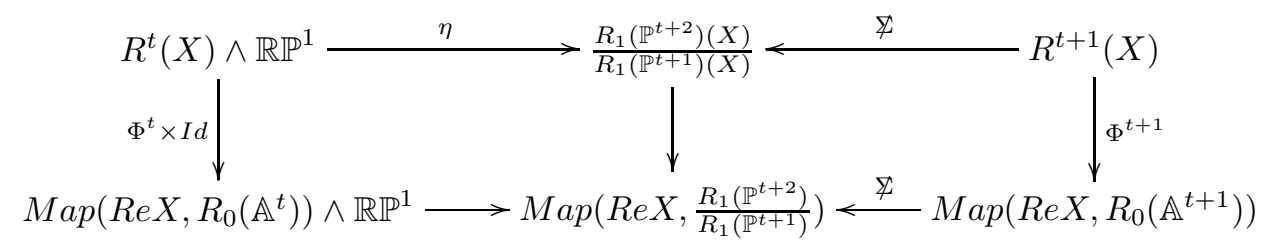

The right arrow in the bottom row sends $f \wedge x$ to the map $(f \wedge x)(y)=f(y) \#((x-$ $\left.\left.x_{\infty}\right)+Z_{0}\left(\mathbb{P}^{1}\right)^{a v}\right)$. It is easy to see that these two squares commute and then take the homotopy groups.

Corollary 7.5 For a real projective variety $X$, define $\mathscr{F}^{t}=\Phi^{t, k}\left(R L^{t} H^{k}(X)\right)$. By the above Proposition, we have a filtration:

$$
\mathscr{F}^{k} \subseteq \mathscr{F}^{k+1} \subseteq \cdots \subseteq H^{k}\left(\operatorname{Re} X ; \mathbb{Z}_{2}\right)
$$

which is analogous to the "topological filtration" defined by Friedlander and Mazur in [10].

\subsection{The H-operator In Reduced Real Morphic Cohomol- ogy}

From the construction of $S$-map, we have a map

$$
H: R^{t}(X) \hookrightarrow R^{t}(X) \times R_{0}\left(\mathbb{P}^{1}\right) \longrightarrow R^{t+1}(X)
$$

and then taking the homotopy groups, we have

$$
H: R L^{t} H^{k}(X) \longrightarrow R L^{t+1} H^{k+1}(X)
$$

which is the $H$-operator in reduced real morphic cohomology. 


\subsection{Natural Transformations From Reduced Real Lawson Homology To Singular Homology}

In Lawson homology, there is a natural transformation

$$
\Phi_{r, k}: L_{r} H_{k}(X) \longrightarrow H_{k}(X ; \mathbb{Z})
$$

defined by iterating the $s$-map in Lawson homology $r$ times and then composite with the Dold-Thom isomorphism. We show that similar construction is valid in reduced real Lawson homology and we get a natural transformation from reduced real Lawson homology to singular homology of the real points with $\mathbb{Z}_{2}$-coefficients.

Fix a point $x_{\infty} \in \mathbb{R} \mathbb{P}^{1}$.

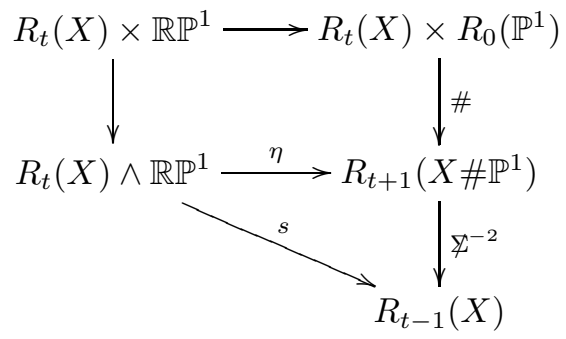

The map on the top horizontal row sends $\left(V+Z_{t}(X)^{a v}, x\right) \in R_{t}(X) \times \mathbb{R P}^{1}$ to $\left(V+Z_{t}(X)^{a v}, x-x_{\infty}+Z_{0}\left(\mathbb{P}^{1}\right)^{a v}\right) \in R_{t}(X) \times R_{0}\left(\mathbb{P}^{1}\right)$. It reduces to a map $\eta$ from the smash product of $R_{t}(X)$ and $\mathbb{R P}^{1}$ to $R_{t+1}\left(X \# \mathbb{P}^{1}\right)$. Finally, we take the homotopy inverse of the suspension map twice. So we get a map $s: \mathbb{S}^{1} \wedge R_{t}(X) \longrightarrow R_{t-1}(X)$ which induces a map in the reduced real Lawson homology

$$
s: R L_{t} H_{k}(X) \longrightarrow R L_{t-1} H_{k}(X) .
$$

We iterate this map $t$ times and then apply the Dold-Thom isomorphism $\tau$ : $\pi_{k} R_{0}(X) \stackrel{\cong}{\longrightarrow} H_{k}\left(\operatorname{Re} X ; \mathbb{Z}_{2}\right)$. This gives us a map

$$
\Phi_{t, k}: R L_{t} H_{k}(X) \longrightarrow H_{k}\left(\operatorname{Re} X ; \mathbb{Z}_{2}\right)
$$

where $\Phi_{t, k}=\tau \circ s^{t}$.

Proposition 7.6 For a morphism $f: X \longrightarrow Y$ between real projective varieties $X, Y$, the following diagram commutes:

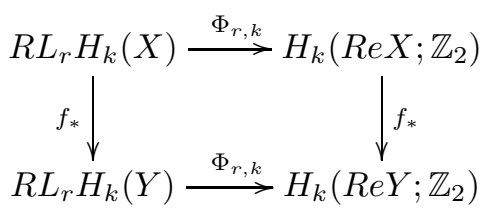

Thus $\Phi$ is a natural transformation from reduced real Lawson homology to singular homology of real points with $\mathbb{Z}_{2}$-coefficients. 
Proof The following diagram commutes:

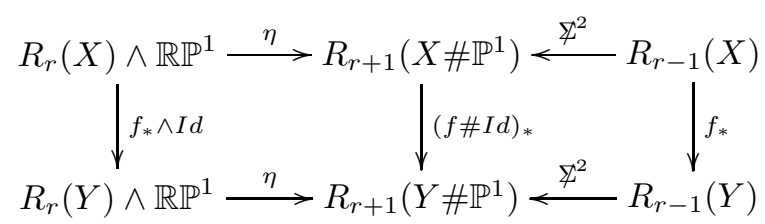

Taking homotopy groups, we have the following commutative diagram of $s$-map

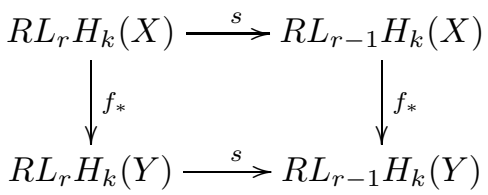

And by the functoriality of Dold-Thom isomorphism, we have the commutative diagram:

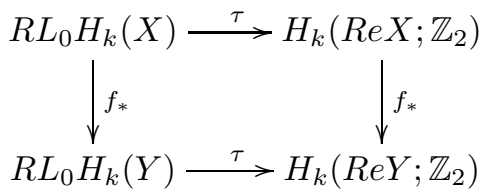

Applying the commutative diagram of $s$-map $r$ times and applying the commutative diagram of Dold-Thom isomorphism, we get the required commutativity.

\subsection{Filtrations}

Definition (The geometric filtration) Let $X$ be a real projective variety and denote by $R G_{j} H_{n}(X) \subset H_{n}\left(R e X ; \mathbb{Z}_{2}\right)$ the subspace of $H_{n}\left(R e X ; \mathbb{Z}_{2}\right)$ generated by the image of maps $H_{n}\left(\operatorname{Re} Y ; \mathbb{Z}_{2}\right) \longrightarrow H_{n}\left(\operatorname{Re} X ; \mathbb{Z}_{2}\right)$ induced from all morphisms $Y \longrightarrow X$ of real projective variety $Y$ of dimension $\leq 2 n-j$. The subspaces $R G_{j} H_{n}(X)$ form a decreasing filtration:

$$
\cdots \subset R G_{j} H_{n}(X) \subset R G_{j-1} H_{n}(X) \subset \cdots \subset R G_{0} H_{n}(X) \subset H_{n}\left(R e X ; \mathbb{Z}_{2}\right)
$$

which is called the geometric filtration.

The $s$-map in reduced real Lawson homology enables us to define a filtration which is analogous to the topological filtration in Lawson homology.

Definition (The topological filtration) Suppose that $X$ is a real projective variety. Let $R T_{t} H_{n}(X)$ denote the subspace of $H_{n}\left(R e X ; \mathbb{Z}_{2}\right)$ given by the image of $\Phi_{t, n}$, i.e.,

$$
R T_{t} H_{n}(X)=\Phi_{t, n}\left(R L_{t} H_{n}(X)\right) .
$$


The subspaces $R T_{t} H_{n}(X)$ form a decreasing filtration:

$$
\cdots \subset R T_{t} H_{n}(X) \subset R T_{t-1} H_{n}(X) \subset \cdots \subset R T_{0} H_{n}(X)=H_{n}\left(R e X ; \mathbb{Z}_{2}\right),
$$

and $R T_{t} H_{n}(X)$ vanishes if $t>n$. This filtration is called the topological filtration.

It was conjectured by Friedlander and Mazur in [10] that the topological filtration and geometric filtration in Lawson homology coincide. We post the similar conjecture for reduced real Lawson homology.

Conjecture For a smooth real projective variety $X$, the topological filtration and the geometric filtration in reduced real Lawson homology coincide, i.e.,

$$
R T_{t} H_{n}(X)=R G_{t} H_{n}(X) .
$$

\subsection{The h-Operator}

Let us now construct a map which is the analogue of the $h$-operator in Lawson homology. Consider the map

$$
h: R_{r}(X) \hookrightarrow R_{r}(X) \times R_{0}\left(\mathbb{P}^{1}\right) \stackrel{\#}{\longrightarrow} R_{r+1}\left(X \# \mathbb{P}^{1}\right) \stackrel{\mathbb{Z}^{-2}}{\longrightarrow} R_{r-1}(X)
$$

which induces a map, called the $h$-operator, in reduced real Lawson homology:

$$
h: R L_{r} H_{k}(X) \longrightarrow R L_{r-1} H_{k-1}(X) .
$$

\subsection{Slant Product}

Suppose that $X, Y$ are projective varieties. Define a product

$$
Z_{r}(Y)(X) \times Z_{p}(X) \longrightarrow Z_{r+p}(Y)
$$

by sending $(f, V)$ to $\operatorname{Pr}_{*}(f \bullet(V \times Y))$ where $\operatorname{Pr}: X \times Y \longrightarrow Y$ is the projection and we consider $f$ as a cycle in $X \times Y$ which intersects $V \times Y$ properly. It is proved in [6, Proposition 7.1] that this is a continuous pairing.

Now suppose that $X, Y$ are real projective varieties. Since in the definition, each operation is real, the above pairing reduces to a pairing

$$
R_{r}(Y)(X) \times R_{p}(X) \longrightarrow R_{r+p}(Y)
$$

and it is easy to see that it reduces again to

$$
R_{r}(Y)(X) \wedge R_{p}(X) \longrightarrow R_{r+p}(Y) .
$$

Therefore, we have a slant product:

$$
R L^{r} H^{k}(X ; Y) \otimes R L_{p} H_{n}(X) \longrightarrow R L_{r+p} H_{n-k+2 r}(Y) .
$$


Fix $r=0, p \leq t$. For $Y=\mathbb{P}^{t-1}$, the product from the above construction sends

$$
R_{0}\left(\mathbb{P}^{t-1}\right)(X) \wedge R_{p}(X) \longrightarrow R_{p}\left(\mathbb{P}^{t-1}\right) .
$$

Therefore, the product

$$
R_{0}\left(\mathbb{P}^{t}\right)(X) \wedge R_{p}(X) \longrightarrow R_{p}\left(\mathbb{P}^{t}\right)
$$

reduces to

$$
\frac{R_{0}\left(\mathbb{P}^{t}\right)(X)}{R_{0}\left(\mathbb{P}^{t-1}\right)(X)} \wedge R_{p}(X) \longrightarrow \frac{R_{p}\left(\mathbb{P}^{t}\right)}{R_{p}\left(\mathbb{P}^{t-1}\right)} .
$$

Since $\frac{R_{p}\left(\mathbb{P}^{t}\right)}{R_{p}\left(\mathbb{P}^{t-1}\right)}=R_{p}\left(\mathbb{A}^{t}\right) \cong R_{0}\left(\mathbb{A}^{t-p}\right)$, we have a pairing:

$$
R^{t}(X) \wedge R_{p}(X) \longrightarrow R_{0}\left(\mathbb{A}^{t-p}\right)
$$

which induces a Kronecker pairing:

$$
R L^{t} H^{k}(X) \otimes R L_{p} H_{k}(X) \longrightarrow \mathbb{Z}_{2}
$$

for $p \leq k \leq t$.

Proposition 7.7 Let $X$ be a real projective variety. Then for all $s \geq q$, the diagram

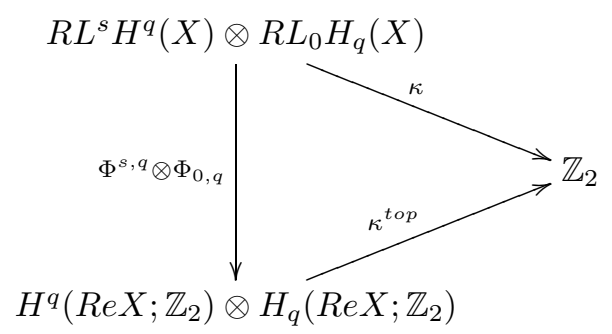

commutes; i.e., under the natural transformation to $\mathbb{Z}_{2}$-singular theory, the Kronecker pairing introduced above is carried to the topological one.

Proof The canonical homeomorphism between $R_{0}(X)$ and $\frac{Z_{0}(\operatorname{Re} X)}{2 Z_{0}(\operatorname{Re} X)}$ is given by sending an element $c \in R_{0}(X)$ to the cycle formed by real points of $X$ that represent $c$ which is unique modulo 2, i.e., there exist unique $x_{1}, \ldots ., x_{k} \in \operatorname{Re} X$ such that $c=\sum_{i=1}^{k} x_{i}+Z_{0}(X)^{a v}$ and $c$ is mapped to $\sum_{i=1}^{k} x_{i}+2 Z_{0}(R e X)$ as the proof in Proposition 2.8. In the following, we will assume that $c$ is represented by real points.

Consider the following diagram:

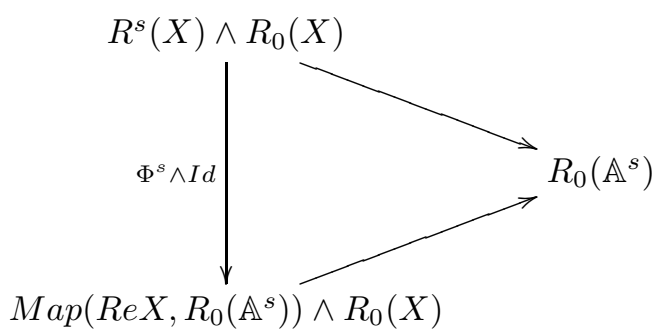


The slant product on the top horizontal arrow sends $\left(f+Z_{0}\left(\mathbb{P}^{s}\right)^{a v}+R_{0}\left(\mathbb{P}^{s-1}\right)(X), \sum_{i} x_{i}+\right.$ $\left.Z_{0}(X)^{a v}\right)$ to $\left(\sum_{i} f\left(x_{i}\right)+Z_{0}\left(\mathbb{P}^{s}\right)^{a v}+R_{0}\left(\mathbb{P}^{s-1}\right)\right)$ and the pairing in the bottom arrow sends $\left(\varphi, \sum_{i} x_{i}\right)$ to $\sum_{i} \varphi\left(x_{i}\right)$. Under the natural maps, it is not difficult to see that the diagram commutes.

Taking homotopy groups, from the bottom horizontal arrow, we get a pairing:

$$
\kappa: H^{q}\left(\operatorname{Re} X ; \mathbb{Z}_{2}\right) \otimes H_{q}\left(\operatorname{Re} X ; \mathbb{Z}_{2}\right) \longrightarrow \mathbb{Z}_{2}
$$

Observe that the following diagram commutes:

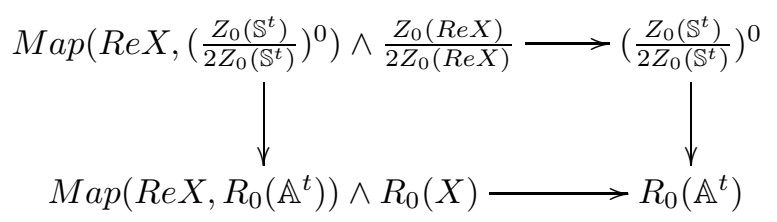

where $\left(\frac{Z_{0}\left(\mathbb{S}^{t}\right)}{2 Z_{0}\left(\mathbb{S}^{t}\right)}\right)^{0}=K\left(\mathbb{Z}_{2}, t\right)$ is the path-connected component of $\frac{Z_{0}\left(\mathbb{S}^{t}\right)}{2 Z_{0}\left(\mathbb{S}^{t}\right)}$ which contains the identity and the canonical quotient map $S^{t} \longrightarrow \mathbb{R P}^{t}$ induces a map from $\left(\frac{Z_{0}\left(\mathbb{S}^{t}\right)}{2 Z_{0}\left(\mathbb{S}^{t}\right)}\right)^{0}$ to $R_{0}\left(\mathbb{A}^{t}\right)$ and hence a map from $\operatorname{Map}\left(\operatorname{Re} X,\left(\frac{Z_{0}\left(\mathbb{S}^{t}\right)}{2 Z_{0}\left(\mathbb{S}^{t}\right)}\right)^{0}\right)$ to $\operatorname{Map}\left(\operatorname{Re} X, R_{0}\left(\mathbb{A}^{t}\right)\right)$. Each vertical arrow of the diagram above is a homotopy equivalence. Thus to prove this Proposition it will suffice to establish the following lemma which is a generalization of Lemma 8.3 in [6].

We use the convention that $\mathbb{Z}_{0}=\mathbb{Z}$ and $\frac{Z_{0}(Y)}{0 Z_{0}(Y)}=Z_{0}(Y)$.

Lemma 7.8 For any finite $C W$-complex $Y$ and $p=0$ or a prime number, the pairing

$$
\operatorname{Map}\left(Y,\left(\frac{Z_{0}\left(\mathbb{S}^{t}\right)}{p Z_{0}\left(S^{t}\right)}\right)^{0}\right) \wedge \frac{Z_{0}(Y)}{p Z_{0}(Y)} \longrightarrow\left(\frac{Z_{0}\left(\mathbb{S}^{t}\right)}{p Z_{0}\left(S^{t}\right)}\right)^{0}
$$

sending $\left(f, \sum_{i} n_{i} y_{i}+p Z_{0}(Y)\right)$ to $\sum_{i} n_{i} f\left(y_{i}\right)+p Z_{0}\left(\mathbb{S}^{t}\right)$ induces a pairing

$$
\kappa: H^{q}\left(Y ; \mathbb{Z}_{p}\right) \otimes H_{q}\left(Y ; \mathbb{Z}_{p}\right) \longrightarrow \mathbb{Z}_{p}
$$

which is the topological Kronecker pairing $\kappa^{\text {top }}$ where $K=\left(\frac{Z_{0}\left(\mathbb{S}^{t}\right)}{p Z_{0}\left(\mathbb{S}^{t}\right)}\right)^{0}=K\left(\mathbb{Z}_{p}, t\right)$ is the component of $\frac{Z_{0}\left(\mathbb{S}^{t}\right)}{p Z_{0}\left(\mathbb{S}^{t}\right)}$ containing the identity.

Proof For a continuous map $\varphi: Y \longrightarrow Z$, from the definition, $\kappa$ has following naturality property:

$$
\kappa\left(\varphi^{*} \alpha, u\right)=\kappa\left(\alpha, \varphi_{*} u\right)
$$

for all $\alpha \in H^{q}\left(Z ; \mathbb{Z}_{p}\right)$ and $u \in H_{q}\left(Y ; \mathbb{Z}_{p}\right)$.

Let $Y=\mathbb{S}^{q}, K=\left(\frac{Z_{0}\left(\mathbb{S}^{t}\right)}{p Z_{0} \mathbb{S}^{t}}\right)^{0}=K\left(\mathbb{Z}_{p}, t\right)$. A generator of $H^{q}\left(\mathbb{S}^{q} ; \mathbb{Z}_{p}\right)=$ $\pi_{t-q} \operatorname{Map}\left(\mathbb{S}^{q}, K\right)$ is given by the homotopy class of $f: \mathbb{S}^{t-q} \longrightarrow \operatorname{Map}\left(\mathbb{S}^{q}, K\right)$ where $f(x)(y)=x \wedge y+p Z_{0}\left(\mathbb{S}^{t}\right)$ and a generator of $H_{q}\left(\mathbb{S}^{q} ; \mathbb{Z}_{p}\right)=\pi_{q}\left(\frac{Z_{0}\left(\mathbb{S}^{q}\right)}{p Z_{0}\left(\mathbb{S}^{q}\right)}\right)$ is 
given by the homotopy class of $g: \mathbb{S}^{q} \longrightarrow \frac{Z_{0}\left(\mathbb{S}^{q}\right)}{p Z_{0}\left(\mathbb{S}^{q}\right)}$ defined by $g(x)=x+p Z_{0}\left(\mathbb{S}^{q}\right)$. Then the map $f \wedge g: \mathbb{S}^{t-q} \wedge \mathbb{S}^{q} \longrightarrow K$ given by

$$
(f \wedge g)(x \wedge y)=f(x)(g(y))=f(x)\left(y+p Z_{0}\left(S^{t}\right)\right)=x \wedge y+p Z_{0}\left(\mathbb{S}^{t}\right)
$$

is the generator of $H_{t}\left(K ; \mathbb{Z}_{p}\right)=\mathbb{Z}_{p}$. Thus $\kappa=\kappa^{\text {top }}$ when $Y$ is a sphere.

Now let $Y=\left(\frac{Z_{0}\left(\mathbb{S}^{q}\right)}{p Z_{0}\left(\mathbb{S}^{q}\right)}\right)^{0}=K\left(\mathbb{Z}_{p} ; q\right)$. Let $i: \mathbb{S}^{q} \longrightarrow Y$ denote the generator of $\pi_{q}(Y)$. Let $u \in H^{q}\left(Y ; \mathbb{Z}_{p}\right)$ and $\sigma \in H_{q}\left(Y ; \mathbb{Z}_{p}\right)$ be given. Since $i_{*}$ : $H_{q}\left(\mathbb{S}^{q} ; \mathbb{Z}_{p}\right) \longrightarrow H_{q}\left(Y ; \mathbb{Z}_{p}\right)$ is an isomorphism, there is an element $\tau \in H_{q}\left(\mathbb{S}^{q} ; \mathbb{Z}_{p}\right)$ with $i_{*} \tau=\sigma$. Then by equation 1 and the case of spheres, $\kappa^{t o p}(u, \sigma)=$ $\kappa^{t o p}\left(i^{*} u, \tau\right)=\kappa\left(i^{*} u, \tau\right)=\kappa(u, \sigma)$.

For a general space $Y$, fix $u \in H^{q}\left(Y ; \mathbb{Z}_{p}\right)$ and let $f: Y \longrightarrow K\left(\mathbb{Z}_{p}, q\right)$ be the map classifying $u$; i.e., $u=f^{*} \iota$ where $\iota \in H^{q}\left(K\left(\mathbb{Z}_{p}, q\right) ; \mathbb{Z}_{p}\right)$ is the canonical generator. Then for any $\tau \in H_{q}\left(Y ; \mathbb{Z}_{p}\right)$, we have

$$
\kappa^{t o p}(u, \tau)=\kappa^{t o p}\left(\iota, f_{*} \tau\right)=\kappa\left(\iota, f_{*} \tau\right)=\kappa(u, \tau) .
$$

This complete the proofs.

\section{The Compatibility Of The Duality Theorem With The $\mathbb{Z}_{2}$-Poincaré Duality}

Let us recall that a projective variety $X$ is said to have full real points if $\operatorname{dim}_{\mathbb{R}} \operatorname{Re} X=\operatorname{dim}_{\mathbb{C}} X$.

Theorem 8.1 Suppose that $X$ is a real projective manifold of dimension $m$ with full real points and $\operatorname{Re} X$ is connected. Then the duality theorem between reduced real morphic cohomology and reduced real Lawson homology is compatible with the $\mathbb{Z}_{2}$-Poincaré duality, i.e., the following diagram commutes:

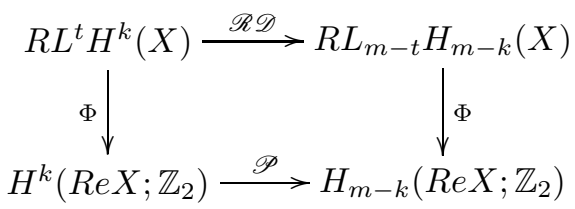

where $\mathscr{P}$ is the Poincaré duality map.

Proof By Proposition [7.4, we reduce the problem to the case $t=m$, i.e., we need to prove the commutativity of the following diagram:

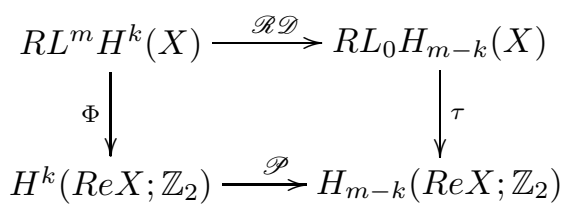


where $\tau$ is the Dold-Thom isomorphism.

Since the evaluation and the intersection products:

$$
\begin{gathered}
H^{k}\left(\operatorname{Re} X ; \mathbb{Z}_{2}\right) \otimes H_{k}\left(\operatorname{Re} X ; \mathbb{Z}_{2}\right) \stackrel{<,>}{\longrightarrow} H_{0}\left(\operatorname{Re} X ; \mathbb{Z}_{2}\right), \\
H_{m-k}\left(\operatorname{Re} X ; \mathbb{Z}_{2}\right) \otimes H_{k}\left(\operatorname{Re} X ; \mathbb{Z}_{2}\right) \stackrel{\odot}{\longrightarrow} H_{0}\left(\operatorname{Re} X ; \mathbb{Z}_{2}\right)
\end{gathered}
$$

are perfect pairings, it suffices to prove that

$$
<\Phi(\alpha), \gamma>=\tau(\mathscr{R} \mathscr{D}(\alpha)) \odot \gamma
$$

for all $\alpha \in R L^{m} H^{k}(X)$ and all $\gamma \in H_{k}\left(R e X ; \mathbb{Z}_{2}\right)$. To prove this equality, it suffices to prove the commutativity of the following diagram:

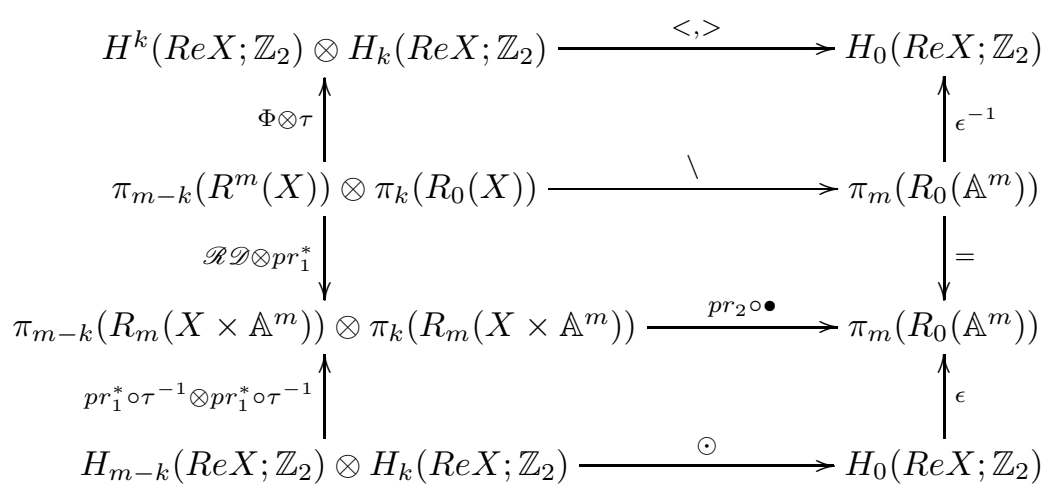

where

$$
\epsilon=p r_{2 *} \circ p r_{1}^{*}: \pi_{0}\left(R_{0}(X)\right) \longrightarrow \pi_{m}\left(R_{0}\left(X \times \mathbb{A}^{m}\right)\right) \longrightarrow \pi_{m}\left(R_{0}\left(\mathbb{A}^{m}\right)\right)
$$

and $\backslash$ is the slant product. The commutativity of the top square follows from the construction of slant product 7.8 and the naturality of Kronecker pairing 7.7.

To verify the commutativity of the middle square of the diagram 2, it suffices to show that the following diagram commutes:

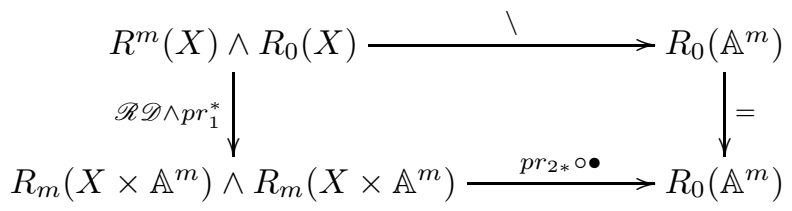

For $f+R_{0}\left(\mathbb{P}^{m-1}\right)(X) \in R^{m}(X), \sum n_{i} x_{i}+Z_{0}(X)^{a v} \in R_{0}(X), \mathscr{R} \mathscr{D}(f+$ $\left.R_{0}\left(\mathbb{P}^{m-1}\right)(X)\right)=f+R_{m}\left(X \times \mathbb{P}^{m-1}\right), p r_{1}^{*}\left(\sum n_{i} x_{i}+Z_{0}(X)^{a v}\right)=\sum n_{i}\left(x_{i} \times\right.$ $\left.\mathbb{P}^{m}\right)+R_{m}\left(X \times \mathbb{P}^{m-1}\right), p r_{2 *}\left[\left(f+R_{m}\left(X \times \mathbb{P}^{m-1}\right)\right) \bullet\left(\sum n_{i}\left(x_{i} \times \mathbb{P}^{m}\right)+R_{m}(X \times\right.\right.$ $\left.\left.\left.\mathbb{P}^{m-1}\right)\right)\right]=p r_{2 *}\left(f \bullet \sum n_{i}\left(x_{i} \times \mathbb{P}^{m}\right)\right)+R_{0}\left(\mathbb{P}^{m-1}\right)$ which is the slant product of $f+R_{m}\left(X \times \mathbb{P}^{m-1}\right)$ and $\sum n_{i} x_{i}+Z_{0}(X)^{a v}$. 
To prove the commutativity of the bottom square, it suffices to prove the commutativity of the following diagram:

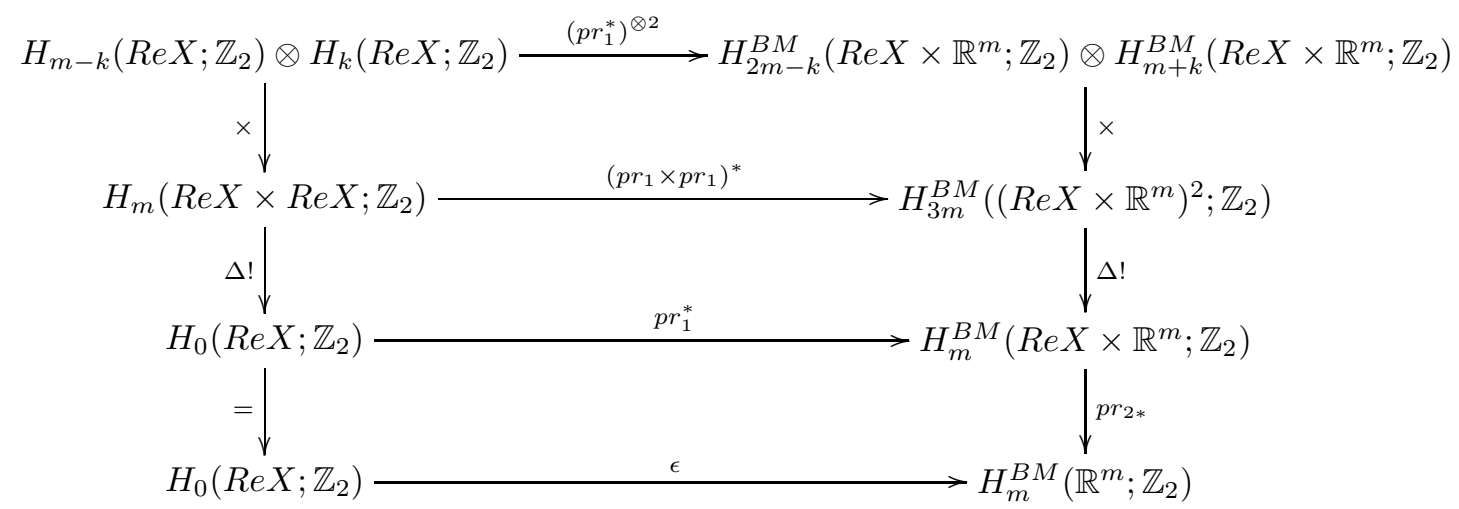

The composition of the maps in the right column can be identified with the map $p r_{2 *} \circ \bullet$ in diagram 2 using the naturality of $\tau$ and the homotopy property of trivial bundle projection of reduced real Lawson homology and the composition of the maps in the left column of diagram 3 is the intersection pairing. Thus the commutativity of diagram 3 implies the commutativity of the last square of 2.

The evident intertwining of the external product $\times$ and the flat pull-back $p r_{1}^{*}$ implies the commutativity of the top square. The Gysin maps and flat pull-backs commute, for a proof, for example in [9, 3.4.d]. These maps are real hence induce maps in reduced real Lawson homology and commute with the Dold-Thom isomorphism. This give the commutativity of the middle square. The commutativity of the bottom square comes from the definition of $\epsilon$.

From the commutative diagram in Theorem 8.1 we have the following result.

Corollary 8.2 If $X$ is a real projective variety of dimension $m$ with full real points and $\operatorname{Re} X$ is connected, then

$$
R L^{t} H^{k}(X) \cong H^{k}\left(\operatorname{Re} X ; \mathbb{Z}_{2}\right), \text { for } 0 \leq t \leq m
$$

\section{A}

We prove that each cycle group that we deal with in this paper has the homotopy type of a CW-complex. The proof is inspired by a result of Friedlander and Walker [11, Proposition 2.5]. Throughout this section, $X, Y$ are projective or real projective varieties of dimension $m$ and $n$ respectively. To simplify our notation, we write $\mathscr{C}_{d}=\mathscr{M} \operatorname{or}\left(X, \mathscr{C}_{r, d}(X)\right)$, the set of morphisms from $X$ to the Chow variety $\mathscr{C}_{r, d}(Y)$, and $\mathscr{C}=\coprod_{d>0} \mathscr{C}_{d}$ the monoid of $Y$-valued $r$-cocycles on $X$. By [6. Lemma 1.4], $\mathscr{C}_{d}$ is a quasi-projective variety. Let $G_{n}=\coprod_{d_{1}+d_{2} \leq n} \mathscr{C}_{d_{1}} \times \mathscr{C}_{d_{2}}$, 
and $K_{n}=G_{n} / \sim$ where $\left(\alpha_{1}, \beta_{1}\right) \sim\left(\alpha_{2}, \beta_{2}\right)$ if and only if $\alpha_{1}+\beta_{2}=\alpha_{2}+\beta_{1}$. The map

$$
\widetilde{i}_{n}: K_{n} \longrightarrow K_{n+1}
$$

induced by the inclusion $i_{n}: G_{n} \rightarrow G_{n+1}$ is an inclusion for each $n$. Let $p_{n}: G_{n} \rightarrow K_{n}$ be the quotient for $n \in \mathbb{N} \cup\{0\}$. Let $G_{n}^{\prime}=p_{n+1}^{-1}\left(\widetilde{i}_{n}\left(k_{n}\right)\right)$, $S_{n}=\coprod_{a+b=n} \mathscr{C}_{a} \times \mathscr{C}_{b}$, and $R_{n}=$ Image $\left\{\coprod_{d_{1}+d_{2}+2 e=n} \mathscr{C}_{d_{1}} \times \mathscr{C}_{d_{2}} \times \mathscr{C}_{e} \rightarrow S_{n}\right\}$ where the map is given by $\left(C_{1}, C_{2}, C_{3}\right) \mapsto\left(C_{1}+C_{3}, C_{2}+C_{3}\right)$. By the proof of [9. Proposition 1.3], $R_{n}$ is a subcomplex of $S_{n}$. Since $G_{n}^{\prime}=G_{n} \cup R_{n}$, hence $G_{n}^{\prime}$ is a CW-complex. Let $\widetilde{K}_{n}=\widetilde{i}_{n}\left(K_{n}\right)$.

Lemma A.1 The diagram

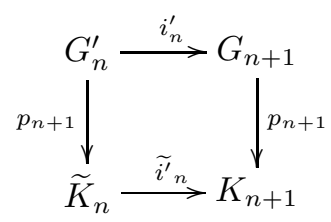

is a diagram of pushout.

Proof Suppose that the following is a commutative diagram:

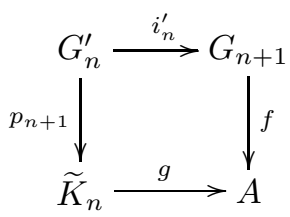

We define a map $\varphi: K_{n+1} \rightarrow A$ by $\varphi(x)=f\left(p_{n+1}^{-1}(x)\right)$. Since for $y \in p_{n+1}^{-1}(x)$ where $x \in \widetilde{K}_{n}, f(y)=g\left(p_{n+1}(y)\right)=g(x)$, and if $x \in K_{n+1}-\widetilde{K}_{n},\left|p_{n+1}^{-1}(x)\right|=1$, hence $f$ is constant on each fibre of $p_{n+1}$. Therefore $\varphi$ is continuous and $f=$ $\varphi \circ p_{n+1}$. It is easy to see that $\varphi \circ{\widetilde{i^{\prime}}}_{n}=g$.

Suppose that $\varphi^{\prime}: K_{n+1} \rightarrow A$ is another map which makes the following diagrams commute:

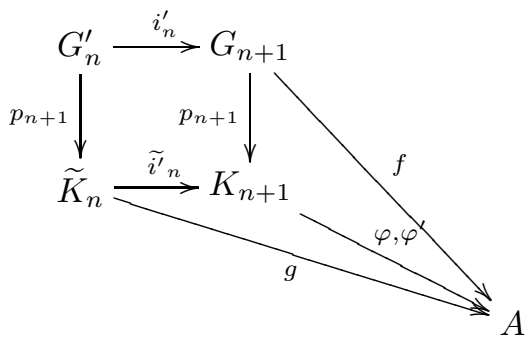

If $x \in \widetilde{K}_{n}, \varphi^{\prime}(x)=g(x)=\varphi(x)$; if $x \in K_{n+1}-\widetilde{K}_{n}$, there is a unique $y \in G_{n+1}-G_{n}^{\prime}$ such that $\varphi^{\prime}(x)=f(y)=\varphi(x)$. Therefore $\varphi=\varphi^{\prime}$. 
Theorem A.2 The cycle space $Z_{r}(Y)(X)$ is a $C W$-complex. In particular, $Z_{r}(X)$ is a $C W$-complex.

Proof We prove by induction. $K_{0}=\{0\}$ is a point. Assume that $K_{n}$ is a $\mathrm{CW}$-complex and the quotient map from $G_{n}$ to $K_{n}$ is regular cellular. Since $\widetilde{K}_{n}$ is homeomorphic to $K_{n}$, and $G_{n}, G_{n}^{\prime}$ are CW-complexes, hence there is a CW-complex $W_{n+1}$ such that the following diagram is a pushout:

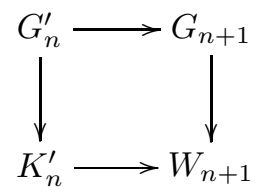

and the map from $K_{n}^{\prime}$ to $W_{n+1}$ is regular cellular. Then $W_{n+1}$ is homeomorphic to $K_{n+1}$, hence $K_{n+1}$ is a CW-complex. And the inclusion map from $K_{n}$ to $K_{n+1}$, the quotient map from $G_{n}$ to $K_{n}$ are regular cellular. Therefore $Z_{r}(Y)(X)$ is a CW-complex.

As proved by Hironaka in [13] that any projective variety admits a triangulation by semi-algebraic simplices which can be chosen so that any specified finite collection of semi-algebraic closed subsets consists of sub-complexes. Recall that a subset in $\mathbb{R}^{n}$ defined by the zero loci of some real polynomials is called a totally real algebraic variety (see [26]). We may embed the projective space $\mathbb{P}^{n}$ into $\mathbb{R}^{N}$ as a totally real algebraic variety ([2, Proposition 3.4.6]), and the conjugation of $\mathbb{P}^{n}$ can be realized as a totally real algebraic map.

Corollary A.3 The $Y$-valued real cycle space $Z_{r}(Y)(X)_{\mathbb{R}}$ is a sub-complex of $Z_{r}(Y)(X)$. In particular, the real cycle group $Z_{r}(X)_{\mathbb{R}}$ is a sub-complex of $Z_{r}(X)$.

Proof The set $\mathscr{C}_{d}(Y)(X)_{\mathbb{R}}$ is the set of real points of $\mathscr{C}_{d}$ which is a totally real quasiprojective variety and thus $\mathscr{C}_{d}(Y)(X)_{\mathbb{R}}$ is a sub-complex of $\mathscr{C}_{d}$. Let $G_{n \mathbb{R}}:=$ $\coprod_{d_{1}+d_{2}=n} \mathscr{C}_{d_{1}}(Y)(X)_{\mathbb{R}} \times \mathscr{C}_{d_{2}}(Y)(X)_{\mathbb{R}}$. Since the quotient map $p_{n}: G_{n} \rightarrow K_{n}$ is regular cellular, hence $K_{n}(X)_{\mathbb{R}}:=p_{n}\left(G_{n \mathbb{R}}\right)$ is a sub-complex of $K_{n}$. Therefore $Z_{r}(Y)(X)_{\mathbb{R}}$ is a sub-complex of $Z_{r}(Y)(X)$.

Corollary A.4 The $Y$-valued averaged cycle space $Z_{r}(Y)(X)^{a v}$ is a sub-complex of $Z_{r}(Y)(X)_{\mathbb{R}}$. In particular, the averaged cycle group $Z_{r}(X)^{\text {av }}$ is a sub-complex of $Z_{r}(X)_{\mathbb{R}}$.

Proof Let $\phi: \mathscr{C}_{d}(Y)(X) \rightarrow \mathscr{C}_{2 d}(Y)(X)$ be the map defined by $\phi(c):=c+\bar{c}$. Since the cycle addition is an algebraic map, and the conjugation is semialgebraic, hence $\phi$ is semi-algebraic. Let $\mathscr{C}_{2 d}(Y)(X)^{a v}$ be the image of $\phi$, then $\mathscr{C}_{2 d}(Y)(X)^{a v}$ is a semi-algebraic set. We may take it as a sub-complex of $\mathscr{C}_{2 d}(Y)(X)_{\mathbb{R}}$. Let $G_{2 n}^{a v}=\bigsqcup_{d_{1}+d_{2}=n} \mathscr{C}_{2 d_{1}}(Y)(X)^{a v} \times \mathscr{C}_{2 d_{2}}(X)^{a v}$. Then $G_{2 n}^{a v}$ is a sub-complex of $G_{2 n \mathbb{R}}$. Therefore $Z_{r}(X)^{a v}$ is a sub-complex of $Z_{r}(X)_{\mathbb{R}}$. 
Theorem A.5 The $Y$-valued reduced real cycle group $R_{r}(Y)(X)$ is a $C W$ complex. In particular, the reduced real cycle group $R_{r}(X)$ is a $C W$-complex.

Proof Let $M_{n}=K_{n}(Y)(X)_{\mathbb{R}}+Z_{r}(Y)(X)^{a v}$. Since $K_{n}(Y)(X)_{\mathbb{R}}$ and $Z_{r}(Y)(X)^{a v}$ are sub-complexes of $Z_{r}(Y)(X)_{\mathbb{R}}$, and the cycle addition + is regular cellular, hence $M_{n}$ is a sub-complex of $Z_{r}(Y)(X)_{\mathbb{R}}$. We have an obvious pushout diagram

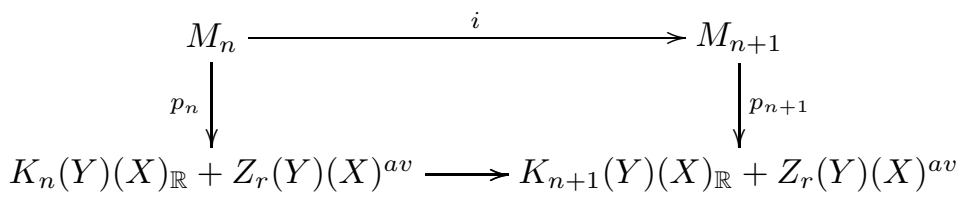

By induction, $K_{n}(Y)(X)_{\mathbb{R}}+Z_{r}(Y)(X)^{a v}$ is a CW-complex, hence $K_{n+1}(Y)(X)_{\mathbb{R}}+$ $Z_{r}(Y)(X)^{a v}$ is homeomorphic to the adjunction space $M_{n+1} \coprod_{p_{n+1}} K_{n}(Y)(X)_{\mathbb{R}}+$ $Z_{r}(Y)(X)^{a v}$ consequently, a CW-complex, and the map from $K_{n}(Y)(X)_{\mathbb{R}}+$ $Z_{r}(Y)(X)^{a v}$ to $K_{n+1}(Y)(X)_{\mathbb{R}}+Z_{r}(Y)(X)^{a v}$ is regular cellular, therefore, $R_{r}(Y)(X)$ is a $\mathrm{CW}$-complex.

Theorem A.6 The reduced real cocycle $R^{t}(X)$ is a $C W$-complex.

Proof Let $K_{n}=\coprod_{d_{1}+d_{2} \leq n} \mathscr{C}_{d_{1}}\left(\mathbb{P}^{t}\right)(X) \times \mathscr{C}_{d_{2}}\left(\mathbb{P}^{t}\right)(X) / \sim, M_{n}=K_{n}+Z_{0}\left(\mathbb{P}^{t}\right)(X)^{a v}+$ $R_{0}\left(\mathbb{P}^{t-1}\right), N_{n}=p_{n}\left(M_{n}\right)$ where $p_{n}$ is the restriction of the quotient map $p$ : $R_{0}\left(\mathbb{P}^{t}\right)(X) \rightarrow R^{t}(X)$ to $M_{n}$. Then we have a pushout diagram

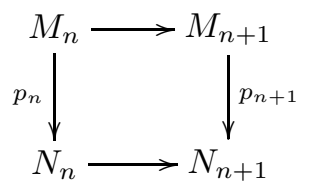

By induction, we show that $R^{t}(X)$ is a $\mathrm{CW}$-complex.

Acknowledgement The author thanks Blaine Lawson for his encouragement and guidance of his study, and for his remarks and corrections of this paper. He also thanks Christian Haesemeyer for his thorough proofreading and the National Center for Theoretical Sciences in Hsinchu, Taiwan for its warm hospitality.

\section{References}

[1] Marcelo Aguilar, Samuel Gitler Carlos Prieto, 'Algebraic Topology from a Homotopical Viewpoint', Universitext, Springer, (2002).

[2] J. Bochnak, M. Coste M.-F. Roy, 'Real Algebraic Geometry', Ergebnisse der Math. und ihrer Grenzgeb. Folge 3, Vol. 36, Berlin Heidelberg, New York, Springer, (1998). 
[3] D. J. Benson, 'Representations and cohomology II: Cohomology of groups and modules', Cambridge Studies in ad. math. 31, (1998).

[4] E. Friedlander, 'Algebraic cycles, Chow varieties, and Lawson homology', Compositio Math. 77 (1991), 55-93.

[5] E. Friedlander, 'Algebraic cocycles on normal, quasi-projective varieties', Compositio Math. 110 (1998), 127-162.

[6] E. Friedlander H.B. Lawson, 'A theory of algebraic cocycles', Annals of Math. 136 (1992), 361-428.

[7] E. Friedlander and H.B. Lawson, 'Moving algebraic cycles of bounded degree', Invent. math. 132 (1998), 91-119.

[8] E. Friedlander and H.B. Lawson, 'Duality relating spaces of algebraic cocycles and cycles', Topology 36 (1997), 533-565.

[9] E. Friedlander and O. Gabber, 'Cycle spaces and intersection theory', Topological Methods in Modern Mathematics (1993), 325-370.

[10] E. Friedlander B. Mazur, 'Filtration on the homology of algebraic varieties', Memoir of the A.M.S., no. 529 (1994).

[11] E. Friedlander M. Walker, 'Function spaces and continuous algebraic pairings for varieties', Compositio Math., 125 (2001), 69-110.

[12] W. Fulton, 'Intersection Theory', Springer-Verlag, New York, (1984).

[13] H. Hironaka, 'Triangulations of algebraic sets', Proc. of Symposia in Pure Math. 29 (1975), 165-185.

[14] F. Hirzebruch, 'Topological methods in algebaic geometry', SpringerVerlag, New York, (1966).

[15] H.B. Lawson, 'Algebraic cycles and homotopy theory', Annals of Math. 129 (1989), 253-291.

[16] H.B. Lawson, 'Cycles and Spectra', Bull Braz Math. Soc. 34, no. 1 (2003), $1-28$.

[17] Lam, 'Spaces of real algebraic cycles and homotopy theory', Stony Brook Phd thesis (1990).

[18] P. Lima-Filho, 'Lawson homology for quasi-projective varieties', Compositio Math. 84 (1992), 1-23.

[19] H.B. Lawson, P. Lima-Filho M. Michelsohn, 'Algebraic cycles and the classical groups I: real cyclces', Topology, 42 (2003), 467-506.

[20] H.B. Lawson, P. Lima-Filho M. Michelsohn, 'On Equivariant Algebraic Suspension', J. Alg. Geo., 7 (1998), 627-650. 
[21] H.B. Lawson M. Michelsohn, 'Algebraic cycles, Bott periodicity, and the Chern characteristic map', The Math. Heritage of Hermann Weyl, Amer. Math. Soc., Providence, (1988), 241-264.

[22] J. Milnor, 'Construction of Universal Bundles, II', The Annals of Math., 63 (1956), 430-436.

[23] Shafarevich, 'Basic Algebraic Geometry', Springer-Verlag, New York, (1977).

[24] M. Walker, 'The morphic Abel-Jacobi map', Compositio Math., 143 (2007), 909-944.

[25] Teh, Jyh-Haur, 'Harnack-Thom Theorem for higher cycle groups and Picard varieties', arXiv.org, math.AG/0509149, (2005).

[26] Teh, Jyh-Haur, 'Complexification of real cycles and the Lawson suspension theorem', J. London Math. Society, 75 (2007), 463-478.

Jyh-Haur Teh

National Tsing Hua University,

Department of Mathematics,

Third General Buidling,

No. 101, Sec 2, Kuang Fu Road, Hsinchu 30043, Taiwan.

Email: jyhhaur@math.nthu.edu.tw 